Contributions to Game Theory and Management, XIV, 127-154

\title{
A Survey on Two Viruses Extensions of Epidemic Model with Continuous and Impulse Control
}

\author{
Elena Gubar and Vladislav Taynitskiy
}

\author{
St. Petersburg State University, \\ 7/9 Universitetskaya nab., Saint Petersburg 199034, Russia, \\ E-mail: e.gubar@spbu.ru, tainitsky@gmail.com
}

\begin{abstract}
The current study represents a survey on several modifications of compartment epidemic models with continuous and impulse control policies. The main contribution of the survey is the modification of the classical Susceptible Infected Recovered (SIR) model with the assumption that two types of viruses are circulating in the population at the same time. Moreover, we also take into consideration the network structure of the initial population in two-virus SIIR models and estimate the effectiveness of protection measures over complex networks. In each model, the optimal control problem has been formalized to minimize the costs of the virus spreading and find optimal continuous and impulse antivirus controllers. All theoretical results are corroborated by a large number of numerical simulations.
\end{abstract}

Keywords: epidemic process, compartment epidemic models, SIR model, optimal control, evolutionary games, virus mutation, structured population.

\section{Introduction}

The current paper represents an overview of the results obtained in the framework of the controlled epidemic models by the authors and their colleagues in (Gubar, 2013; Taynitskiy, 2016b; Taynitskiy, 2017a; Taynitskiy, 2017b). Infection diseases remain a serious medical problem all around the world for many centuries with million deaths per year. The emergence of new diseases such as the severe acute respiratory syndrome (SARS) and, most recently, the rise of Ebola and Zika viruses, Hepatitis, COVID-19 and other diseases represent a few examples of the serious problems that the public health and medical science research need to investigate. A compartment epidemic models provide an appropriate mathematical tool to study the factors that impact on epidemic growth to improve existing treatments and evaluate new effective prevention measures. Moreover, the process of propagation of biological virus in human or animal populations remain the propagation of computer viruses or information/rumors in computer, and social networks. According to this reason spreading processes in different domains can be modelled by various modifications of the Susceptible-Infected-Recovered (SIR) epidemic model. In our review, we will use the term virus to define the object of spreading and population to define the domain of spreading. In traditional epidemiological compartment models, differential equations are used to capture the dynamic evolution of different classes of host populations. In particular, susceptible (S) is the class of people who are not infected; infected (I) is the class of people who have the disease; and removed or recovered $(\mathrm{R})$ represents the quarantined or immune population. The commonly used SIR model (Capasso, 1993; Conn, 2006) is used to describe the population migrations between these three classes of models.

More specifically, in the current survey, it is assumed that two types of viruses with different strains and fitness functions circulate at the same time in the entire

https://doi.org/10.21638/11701/spbu31.2021.12 
population. Such situation is a natural extension of the epidemic models, as well as, it is known that during the epidemic season several respiratory diseases attack the population. According to this assumption, it is supposed that both types of viruses spread in the populations, and, hence, during an epidemic, different parts of the population are infected. Similar arguments can be applied to the virus propagation process in computer and social networks. In our work, the infected subpopulations are split into two subgroups and we considered a modified SIR model. Therefore, the epidemic process in the total population depends on changes in the virus subpopulation.

One of the main questions in epidemic modelling is how to prevent the rise of epidemics and protect the population from the virus intervention. The application of the optimal control approach helps to answer this question. Formulation of the optimal control problem allows to find the optimal treatment or preventive strategies which protect the population and evaluate the costs of the selected measures.

In the current review, we merge the results, received for several modifications of the epidemiological model with two viruses. The paper is organized as follows. In Section 6. a control-theoretic model to design disease control strategies through quarantine and immunization is formulated to mitigate the impact of epidemics on the entire population. Virus transmission in epidemics can be described by dynamics on a graph where vertices denote individuals and an edge connecting a pair of vertices indicates interaction between individuals. Due to a large population of people involved in the process of disease transmission, random graph models such as the small-world networks in (Strogatz, 2001) or scale-free networks in (Barabasi, 1999) are convenient to capture the heterogeneous patterns in the large scale complex network. We study two variants of controlled multi-strain epidemic models for heterogeneous populations over a large complex network. One is the Susceptible-InfectedRecovered (SIR) epidemic process, where the control is to quarantine a fraction of the infected individuals. Another model is the Susceptible-Infected-Susceptible (SIS) epidemic process, here we assume that the control corresponds to treatment of the infected individuals, however, treated individuals can become susceptible again to the infection of the disease with the course of time.

In Section 9. the virus control mechanism is to remove an optimal fraction of the infected items at discrete points in time. Such mechanism is also known as an optimal impulse controller. The hybrid nature of discrete-time control policy of continuous-time epidemic dynamics together with the network structure poses a challenging optimal control problem. We apply the modified Pontryagin's maximum principle for impulsive systems to obtain an optimal structure of the controller and corroborate it by using numerical experiments to demonstrate the structure of the optimal control and the controlled dynamics. This work extends the investigation of previous related works (Gubar, 2013; Taynitskiy, 2017a; Taynitskiy, 2017b) to a new paradigm of coupled epidemic models and the regime of optimal impulsive control. In Section 2., we discuss related work to our model. The paper is concluded in Section 11..

\section{Related works}

The recent literature has seen a surge of interest in using optimal control and game-theoretic methods to study disease control of respiratory viruses for public health (Newman, 2005; Bomze, 2011; Vespignani, 2015). This research problem has 
been arisen from the work (Kermack, 1927), where a SIR mathematical framework was proposed to study epidemic spread in a homogeneous population. Future research (Perkins, 2020; Rowthorn, 2020) extends the classical model and design a class of compartment epidemic models. Later, in (Wickwire, 1975), a control problem was formulated for a model of the carrier-borne epidemic model, and it was shown that the optimal-control effort switches at maximum once between the maximum and the minimum control effort. In (Behncke, 2000), and (Pappas, 2016) many variants of optimal-control models of SIR epidemics were investigated for different domains: vaccination and health-promotion campaigns, information security, information spreading, etc. For example, in (Francis, 2004; Butler, 2012), a dynamic SIR epidemic model was used to identify the optimal vaccination-policy mixes for flu season.

Epidemic models have also been used in computer science and engineering to describe the evolution of worm propagation in computer networks (Khouzani, 2011; Taynitskiy, 2016a; Newman, 2016). In (Altman, 2010; Khouzani, 2010), optimalcontrol methods were used to study the class of epidemic models in mobile wireless networks, and Pontryagin's maximum principle was used to quantify the damage that virus could inflict on the network by deploying optimum decision rules.

Game-theoretic approaches were also used to analyze the strategic interactions between malicious worms and the system under attack. In (Omic, 2009), a virusprotection game was proposed based on two-state epidemic models for $N$ nodes and the characterization of the equilibrium focus on the steady-state of the response. In (Zhu, 2012), static- and dynamic-game frameworks were used to design equilibriumrevocation strategies for defending sensor networks from node-capturing and cloning attacks. It was shown that the $N+1$ non-zero-sum differential game framework was equivalent to a zero-sum differential game between a team of $N$ attackers and the system. In (Fu, 2011; Taynitskiy, 2015) the SIR model was combined with a game-theoretical approach to define the optimal medical approach: vaccination or treatment of seasonal influenza.

In many modern studies, different modifications of SIR models were used to estimate the behaviour of infectious diseases, such as Ebola and Severe acute respiratory syndrome (SARS) (Chowell, 2017). Nowadays many research projects have concentrated on the study of the pandemic outbreak, generated by the spreading of SARS-COV-2 all over the world. Many research focuses not only on medical aspects of the coronavirus propagation but also on the economic effects of the application of long and short-terms lockdown (Pragyan, 2020; Rowson, 2020).

Different from recent research, this survey considers a novel framework based on the SIR epidemic model including virus mutations. This framework is motivated by the fact that the epidemic spread of a virus can facilitate virus mutations or parallel spreading, strengthening its virulence, which, in turn, accelerates the spread and deteriorates epidemics.

\section{Epidemic process for simultaneously spreading viruses}

Infectious diseases, such as influenza, SARS, and novel coronavirus, are urgent public health problems in modern urban environments. These infections spread faster, especially in large urban populations, and affects people's lifestyle and working facilities. The appearance of epidemics depends on many factors, such as the size of the human population, and the virus strain and virulence, and it has be- 
come important to use effective tools to reduce their impact on human populations (Bonhoeffer, 1997; Gjorgjieva, 2005; Fu, 2011).

In this section of the survey, we present a joint dynamic process of virus propagation and its impact on human populations, which is defined by extended SIIR (Kermack, 1927; Khatri, 2003; Gubar, 2013) epidemic model. Based on the standard approach, a human population of size $N$ is divided into subpopulations: the susceptible, the infected, and the recovered. However, it is assumed that the virus has two types or strains, denoted by $V_{1}$ and $V_{2}$, and without loss of generality, we assume that $V_{2}$ dominates virus $V_{1}$. A virus with higher virulence has a higher probability of infection. The fitness of virus type $V_{i}$ in the population is $\bar{J}_{i}\left(V_{i}, V_{j}\right), i, j=1,2$, which depends on the survivability of the virus among its infected population (e.g., human beings).

According to this assumption, a human population is split into four groups: the susceptible, the infected by virus $V_{1}$, the infected by virus $V_{2}$, and the recovered. The Susceptible $(S)$ are a subpopulation of human beings that are not infected by viruses but could be infected by one or both types of viruses, and they do not have immunity to them. We assume that two types of viruses coexist at the same time. Human organisms can be occupied by both types of viruses, and, hence, this leads to competition between viruses for the host. Depending on virus strength, people infected by the first virus $\left(I_{1}\right)$ or by the second virus $\left(I_{2}\right)$ belong to the Infected subpopulation. The Recovered subpopulation $(R)$ consists of people recovered from being infected. The mixing of urban populations allows viruses to spread quickly, and each person in the population is assumed to be in contact with others with equal probability. Hence, when a susceptible individual interacts with an infected, the virus spreads.

\section{SIIR-model with continuous control}

In the paper (Gubar, 2013) we modeled a virus spreading in an urban population using the epidemiological SIIR model, coupled with virus mutation process. A system of differential equations was used to describe the fraction of the urban population as a function of time. At the time moment $t$, the fractions of the population who are susceptible, infected by virus $V_{1}$, infected by virus $V_{2}$, and recovered are equal to $n_{S}(t), n_{I_{1}}(t), n_{I_{2}}(t), n_{R}(t)$, respectively. Also condition $N=n_{S}(t)+n_{I_{1}}(t)+n_{I_{2}}(t)+n_{R}(t)$ is satisfied. Define the fraction of susceptible as $S(t)=\frac{n_{S}(t)}{N}$, the fractions of infected as $I_{1}(t)=\frac{n_{I_{1}}(t)}{N}, I_{2}(t)=\frac{n_{I_{2}}(t)}{N}$, and $R(t)=\frac{n_{R}(t)}{N}$ are the fraction of recovered. At the beginning of epidemic process at $t=0$, most people are in the Susceptible state, small groups in the total population are infected, and the other people are in the recovered subpopulation. Hence, the initial states are: $0<S(0)=S^{0}<1,0<I_{1}(0)=I_{1}^{0}<1,0<I_{2}(0)=I_{2}^{0}<1$, $R(0)=1-S^{0}-I_{1}^{0}-I_{2}^{0}$.

The extended the SIIR model to describe the process of propagation:

$$
\begin{aligned}
& \frac{d S}{d t}=-\delta_{1}(t) S(t) I_{1}(t)-\delta_{2}(t) S(t) I_{2}(t) ; \\
& \frac{d I_{1}}{d t}=\left(\delta_{1}(t) S(t)-\sigma_{1}-u_{1}(t)\right) I_{1}(t) ; \\
& \frac{d I_{2}}{d t}=\left(\delta_{2}(t) S(t)-\sigma_{2}-u_{2}(t)\right) I_{2}(t) ; \\
& \frac{d R}{d t}=\left(\sigma_{1}+u_{1}(t)\right) I_{1}(t)+\left(\sigma_{2}+u_{2}(t)\right) I_{2}(t) ;
\end{aligned}
$$


where $\delta_{i}(t)$ are the infection rates for virus $V_{i}, i=1,2$ at the time moment $t$. Virus mutation leads to changes in the speed of the virus spreading. Parameters $\sigma_{1}$ and $\sigma_{2}$ are self-recovery rates of viruses $V_{1}$ and $V_{2}$. They show the probability that infected nodes from subgroups $I_{1}$ or $I_{2}$ are recovered from the infection without causing any costs to our system. Infection rate is defined as the product of infection transmissibility, i.e., the probability of infection being transmitted during contact:

$$
\delta_{i}(t)=\delta_{i}(t-\Delta t)+\delta_{i}(t-\Delta t)\left(I_{i}(t)-I_{i}(t-\Delta t)\right)
$$

where $\delta_{i}(0)=\delta_{i_{0}} I_{i}(0)$. Here $\delta_{i_{0}}, i=1,2$, determine the virulence of the particular virus or the ability to infect a susceptible host.

From the previous research, it is known, that spreading of viruses can be controlled using prevention measures such as medical treatment or isolation of infected individuals of the population. Medical treatment or quarantine isolation reduces the number of infected individuals in the urban population. These prevention measures can be interpreted as control parameters in the system defined as $u=\left(u_{1}, u_{2}\right)$. Here, $u_{i}$ are fractions of the infected that are quarantined or under intensive medical treatment: $0 \leq u_{1}(t) \leq 1,0 \leq u_{2}(t) \leq 1$, for all $t$.

\subsection{Objective Function}

In this work, we minimize aggregated cost in time interval $[0, T]$. At any given $t$, the following costs exist in the system: $f_{1}\left(I_{1}(t)\right), f_{2}\left(I_{2}(t)\right)$ are infected costs; $g(R(t))$ is benefit rate function; $h_{1}\left(u_{1}(t)\right), h_{2}\left(u_{2}(t)\right)$ are costs for medical treatments (i.e., quarantine or removal) that help reduce the epidemic spread; $k_{I_{1}}, k_{I_{1}}, k_{R}$, costs and benefit for invective and recovered at the end of the epidemic. Here, functions $f_{i}\left(I_{i}(t)\right)$ are non-decreasing and twice-differentiable, convex functions, i.e., $f_{i}(0)=0, f_{i}\left(I_{i}(t)\right)>0$ for $I_{i}(t)>0, i=1,2 ., g(R(t))$ is non-decreasing and differentiable function and $g(0)=0, h_{i}\left(u_{i}(t)\right)$ is twice-differentiable and increasing function in $u_{i}(t)$ such as $h_{i}(0)=0, h_{i}\left(u_{i}(t)\right)>0, \quad i=1,2$, when $u_{i}(t)>0$. We represent the structure of the optimal control strategies for the general case of costs functions. Hence, in particular cases this condition will be satisfied for any functions with the same properties.

The cost for the aggregated system is given by

$$
J=\int_{0}^{T} f_{1}\left(I_{1}(t)\right)+f_{2}\left(I_{2}(t)\right)-g(R(t))+h_{1}\left(u_{1}(t)\right)+h_{2}\left(u_{2}(t)\right) d t+
$$

and the optimal-control problem is to minimize the cost, i.e., $\min _{\left\{u_{1}, u_{2}\right\}} J$. To simplify the analysis, we consider the case where $k_{I_{1}}=k_{I_{1}}=k_{R}=0$.

\subsection{Optimal Control of SIIR-model with continuous control}

We used Pontryagin's maximum principle (Pontryagin, 1987) to find optimal control $u(t)=\left(u_{1}(t), u_{2}(t)\right)$ to the problem described in Section 4.1. above. Define associated Hamiltonian $H$ and adjoint functions $\lambda_{S}(t), \lambda_{I_{1}}(t), \lambda_{I_{2}}(t), \lambda_{R}(t)$ as follows:

$$
\begin{aligned}
H= & f_{1}\left(I_{1}(t)\right)+f_{2}\left(I_{2}(t)\right)-g(R(t))+h_{1}\left(u_{1}(t)\right)+h_{2}\left(u_{2}(t)\right)+ \\
& \left(\lambda_{I_{1}}-\lambda_{S}\right) \delta_{1} S I_{1}+\left(\lambda_{I_{2}}-\lambda_{S}\right) \delta_{2} S I_{2}+\left(\lambda_{R}-\lambda_{I_{1}}\right) \sigma_{1} I_{1}+ \\
& \left(\lambda_{R}-\lambda_{I_{2}}\right) \sigma_{2} I_{2}-\left(\lambda_{I_{1}}-\lambda_{R}\right) I_{1} u_{1}-\left(\lambda_{I_{2}}-\lambda_{R}\right) I_{2} u_{2} .
\end{aligned}
$$


Here, we used condition $R(t)=1-S(t)-I_{1}(t)-I_{2}(t)$. We constructed an adjoint system as follows:

$$
\begin{aligned}
& \dot{\lambda}_{S}(t)=-\frac{\partial H}{\partial S}=-\lambda_{S}\left(-\delta_{1} I_{1}-\delta_{2} I_{2}\right)-\lambda_{I_{1}} \delta_{1} I_{1}-\lambda_{I_{2}} \delta_{2} I_{2} ; \\
& \dot{\lambda}_{I_{1}}(t)=-\frac{\partial H}{\partial I_{1}}=-f_{1}^{\prime}\left(I_{1}\right)+\lambda_{S} \delta_{1} S-\lambda_{I_{1}}\left(\delta_{1} S-\sigma_{1}\right)-\lambda_{R} \sigma_{1} ; \\
& \dot{\lambda}_{I_{2}}(t)=-\frac{\partial H}{\partial I_{2}}=-f_{2}^{\prime}\left(I_{2}\right)+\lambda_{S} \delta_{2} S-\lambda_{I_{2}}\left(\delta_{2} S-\sigma_{2}\right)-\lambda_{R} \sigma_{1} ; \\
& \dot{\lambda}_{R}(t)=-\frac{\partial H}{\partial R}=g^{\prime}(R) ;
\end{aligned}
$$

with transversality conditions given by:

$$
\lambda_{I_{1}}(T)=0, \lambda_{I_{2}}(T)=0, \lambda_{S}(T)=0, \lambda_{R}(T)=0 .
$$

Consider next derivatives:

$$
\begin{aligned}
& \frac{\partial H}{\partial u_{1}}=h_{1}^{\prime}\left(u_{1}\right)-\left(\lambda_{I_{1}}-\lambda_{R}\right) I_{1} ; \\
& \frac{\partial H}{\partial u_{2}}=h_{2}^{\prime}\left(u_{2}\right)-\left(\lambda_{I_{2}}-\lambda_{R}\right) I_{2} .
\end{aligned}
$$

According to Pontryagin's maximum principle, there exist continuous and piecewise continuously differentiable costate functions $\lambda$ that, at every point $t \in[0, T]$, where $u_{1}$ and $u_{2}$ are continuous, satisfy system (5) and Equation (6). In addition, we have:

$$
\left(u_{1}, u_{2}\right) \in \arg \min _{\bar{u}_{1}, \bar{u}_{2} \in[0,1]} H\left(\bar{\lambda},\left(S, I_{1}, I_{2}, R\right),\left(\bar{u}_{1}, \bar{u}_{2}\right)\right) .
$$

We define switching functions $\varphi_{i}$ as follows:

$$
\varphi_{1}(t)=\left(\lambda_{I_{1}}(t)-\lambda_{R}(t)\right) I_{1}(t), \quad \varphi_{2}(t)=\left(\lambda_{I_{2}}(t)-\lambda_{R}(t)\right) I_{2}(t) .
$$

Based on previous research (Pontryagin, 1987; Khouzani, 2010), in this subsection we show that optimal control $u(t)=\left(u_{1}(t), u_{2}(t)\right)$ has the following structural results:

Functions $h_{i}(\cdot)$ and the Hamiltonian are concave

Let $h_{i}(\cdot)$ be a concave functions $\left(h_{i}^{\prime \prime}(\cdot)<0\right)$, then according to $(4)$, the Hamiltonian is a concave function of $u_{i}, i=\overline{1,2}$. There are two different options for $u_{i} \in[0,1]$ that minimize the Hamiltonian, i.e., if at the time moment $t$

$$
h_{i}(0)-\varphi_{i}(t) \cdot 0<h_{i}(1)-\varphi_{i}(t) \cdot 1,
$$

or

$$
h_{i}(1)>\varphi_{i}(t),
$$

then optimal control is $u_{i}=0$ (see Fig. 2 (left)); otherwise $u_{i}=1$ (see Fig. 2 (right)).

For $i=\overline{1,2}$, the optimal control parameters $u_{i}(t)$ are defined as follows:

$$
u_{i}^{*}(t)= \begin{cases}0, & \text { for } \quad \varphi_{i}(t)<h_{i}(1) \\ 1, & \text { for } \quad \varphi_{i}(t) \geq h_{i}(1)\end{cases}
$$



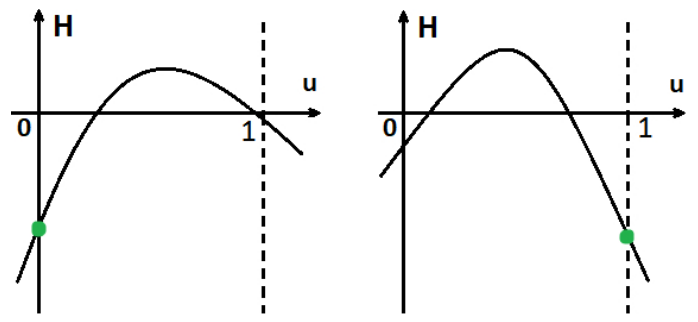

Fig. 1. Hamiltonian if functions $h_{i}(\cdot)$ are concave.

Functions $\varphi_{i}$ are non-negative functions, then there can be at most one time moment $t \in[0, T]$ at which $\varphi_{i}(t)=h_{i}^{\prime}\left(u_{i}(t)\right)$. Moreover, if such moment exists, for example, $t_{1}$, then $\varphi_{i}(t)<h_{i}(1)$ on $0 \leq t<t_{1}$ and $\varphi_{i}(t) \geq h_{i}(1)$ on $t_{1} \leq t<T$.

Functions $h_{i}(\cdot)$ and the Hamiltonian are strictly convex

Let $h_{i}(\cdot)$ be a strictly convex functions $\left(h_{i}^{\prime \prime}(\cdot)>0\right)$, then Hamiltonian is a convex function. Consider the following derivative:

$$
\left.\frac{\partial}{\partial x}\left(h_{i}(x)-\varphi_{i} x\right)\right|_{x=x_{i}}=0,
$$

where $x_{i} \in[0,1], u_{i}^{*}(t)=x_{i}$. There are three different types of points at which the Hamiltonian reaches its minimum (Fig. 3). To find them, we need to consider the derivatives of the Hamiltonian at $u_{i}=0$ and $u_{i}=1$. If the derivatives (10) at $u_{i}=0$ are increasing $\left(h_{i}^{\prime}(0)-\varphi_{i} \geq 0\right)$, then the value of the control that minimizes the Hamiltonian is less than 0 , and according to our restrictions $\left(u_{i} \in[0,1]\right)$ optimal control will be equal to 0 (Fig. 3a). If the derivatives at $u_{i}=1$ are non-increasing $\left(h_{i}^{\prime}(1)-\varphi_{i}<0\right)$, it means that the value of the control that minimizes the Hamiltonian is greater than 1 . Hence the optimal control will be 1 (Fig. 3c); otherwise, we can find such value $u_{i}^{*} \in(0,1)$ (see Fig. $3 \mathrm{~b}$ ).

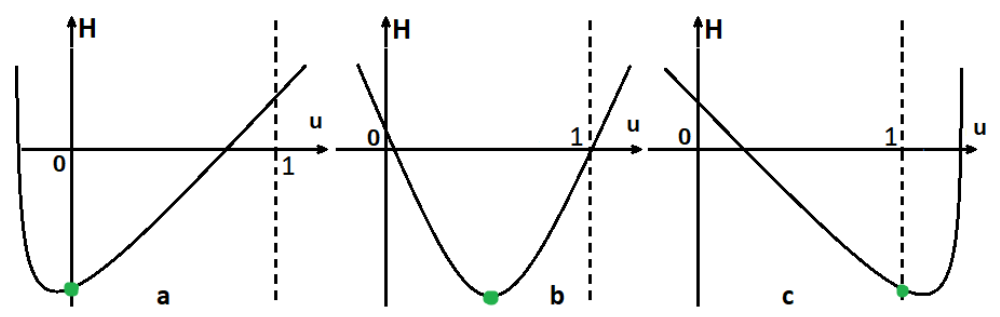

Fig. 2. Hamiltonian when functions $h_{i}(\cdot)$ are convex.

Functions $\varphi_{i}(t), h_{i}^{\prime}(t), u_{i}^{*}(t)$ are continuous at all $t \in[0, T]$. In this case $h_{i}$ is strictly convex and $h_{i}^{\prime}$ is strictly increasing functions, so $h_{i}^{\prime}(0)<h_{i}^{\prime}(1)$. Thus there exist points $t_{0}$ and $t_{1}\left(0<t_{0}<t_{1}<T\right)$ so that conditions (11) are satisfied, and according to $\varphi_{i}(t)$ are non-positive functions. That is:

$$
u_{i}^{*}(t)= \begin{cases}0, & \text { for } \varphi_{i}(t) \leq h_{i}^{\prime}(0) \\ h^{\prime-1}\left(\varphi_{i}\right), & \text { for } h_{i}^{\prime}(0)<\varphi_{i}(t) \leq h_{i}^{\prime}(1) \\ 1, & \text { for } h_{i}^{\prime}(1)<\varphi_{i}(t)\end{cases}
$$




\section{Numerical Simulation of SIIR-model with continuous control}

In this section, we present numerical simulations to corroborate our theoretical results. Consider a city with population $N=100,000$ people, where two viruses, of different strengths, spread $\left(\delta_{1_{0}}=0.4\right.$ and $\left.\delta_{2_{0}}=0.5\right)$. At time moment $t=0$, half of the population are susceptible to the infection, i.e., $S(0)=0.5$. The initial Recovered subpopulation is $R(0)=0$. We set $18 \%$ of population as infected by virus $V_{1}$, and $32 \%$ of the population are infected by virus $V_{2}$, i.e., $I_{1}(0)=0.18$ and $I_{2}(0)=0.32$. The epidemic lasted for 45 days. We assumed that, in this experiment, the self-recovery rates are equal to $\sigma_{1}=0.001$ and $\sigma_{2}=0.002$. During the epidemic, people from the infected population incur infected costs and, hence, we defined cost functions as $f_{1}\left(I_{1}(t)\right)=5 I_{1}(t), f_{2}\left(I_{2}(t)\right)=6 I_{2}(t)$; the benefit rate from the recovered subpopulation is $g(R(t))=0.1 R(t)$. In Section 4.2., we have shown that medical-treatment cost functions to describe the value of treatment can be chosen as concave or strictly convex. In our simulations, or concave cost functions, we used $h_{1}\left(u_{1}(t)\right)=7 u_{1}(t)$, and $h_{2}\left(u_{2}(t)\right)=9 u_{2}(t)$; for convex cost functions, we used $h_{1}\left(u_{1}(t)\right)=15 u_{1}^{2}(t)$ and $h_{2}\left(u_{2}(t)\right)=10 u_{2}^{2}(t)$. The costs here were measured in the same monetary units (m.u.), which could be US dollars, Chinese RMB, or Euros, depending on the context.
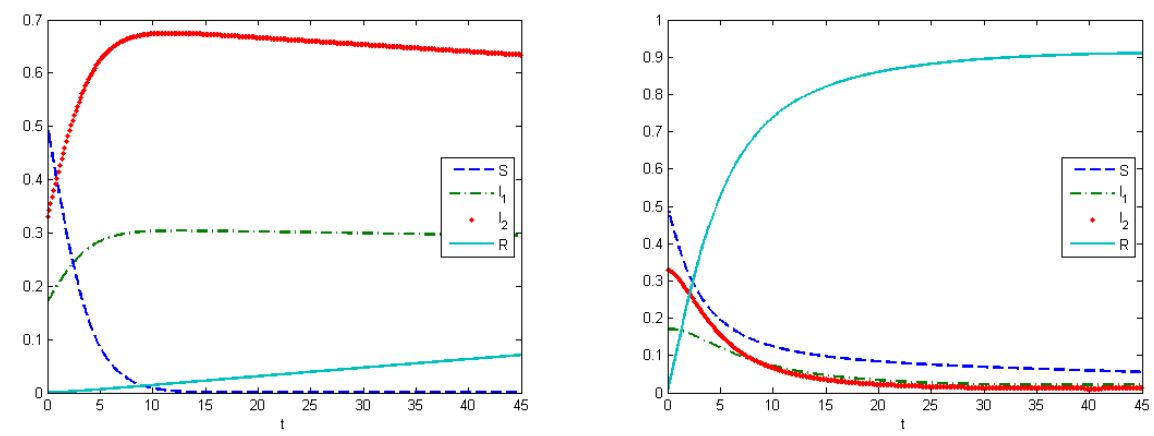

Fig. 3. Experiment 1.1. Left: SIIR model without virus mutation and without applying control. Initial states are $I_{1}(0)=0.18, I_{2}(0)=0.32$, maximum values are $I_{1 \max }=0.3031$ and $I_{2 \max }=0.6742$. Epidemic peaks a reached at 11th and 10th days. Right: SIIR model without virus mutation with application of control. Vertical axes show the fractions of the subpopulations.

Experiment 1.1 shows the behaviour of the system without virus mutation. As a control strategy, we used the medical treatment of the infected host, and the convex form of cost functions, i.e., $h_{1}\left(u_{1}\right)=15 u_{1}^{2}$ and $h_{2}\left(u_{2}\right)=10 u_{2}^{2}$. After simulations, we obtained that the maximum amount of replicas in the case without applying treatment were $I_{1}\left(t_{1}\right)=0.3031$ at $t_{1}=11.25$, and $I_{2}\left(t_{2}\right)=0.6742$ at $t_{2}=10.25$ (Figure 3). From this experiment, we can see that, in the absence of treatment at the end of the epidemic period, our population had the following distribution of Infected hosts: $29 \%$ are infected by the first type of virus and $64 \%$ are infected by the second type of virus. As we have self-recovery rate $\sigma_{1}=0.001$ and $\sigma_{2}=0.002$, a fraction of the Recovered is $R(T)=0.07$. In the case when we applied the control, the fraction of infected hosts is zero at the end of interval $T=45$, and the fraction of the recovered is $92 \%$. There are also some susceptible 
nodes $(S(T)=0.07)$ that were not affected by the epidemic. The aggregated system costs are: $J=251.25$ in the uncontrolled case and $J=67.08$ in the controlled case (Figure 4).
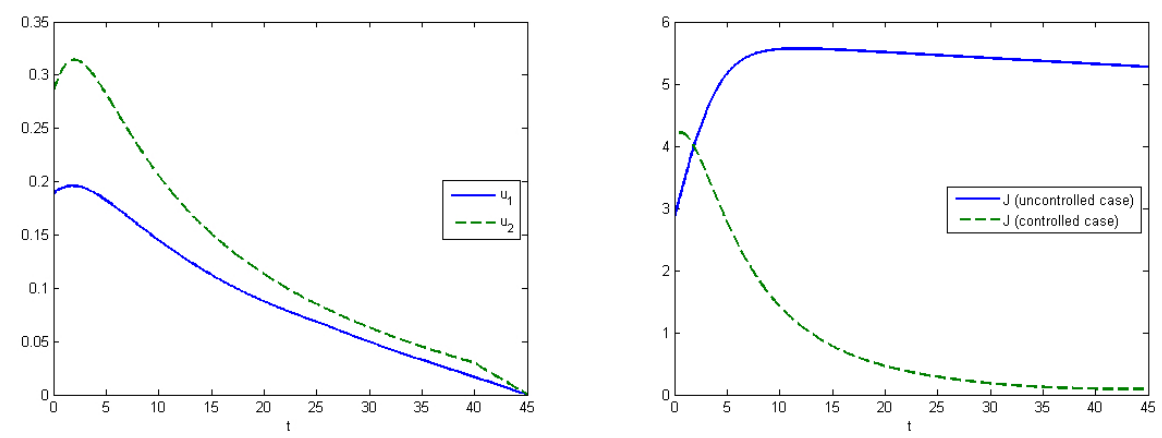

Fig. 4. Experiment 1.1. Left: Optimal control in SIIR model without virus mutation; cost functions are convex $h_{i}\left(u_{i}\right)$. Vertical axis shows the amount of applied control. Right: Comparison of aggregated costs of SIIR model without virus mutation (Controlled case: $J=67.08$; uncontrolled case: $J=251.25$ ). Vertical axis shows the aggregated costs at time moment $t$ in m.u.

Experiment 1.2 represents the SIIR model with virus mutation. Infection rates $\delta_{i}(t), i=1,2$ indicate the speed of viruses spreading in the population. In our work, it means that we take the competition between viruses for the host into account. Here, we supposed that the stronger virus captures more hosts. The strength of the virus depends on the infection rates, which change under a mutation process. In this experiment, infection rates changed by the formula (2). We can allocate available resources in such way that the aggregated costs of the system are minimized. Using the SIIR model, we illustrate how the system develops under various types of control.
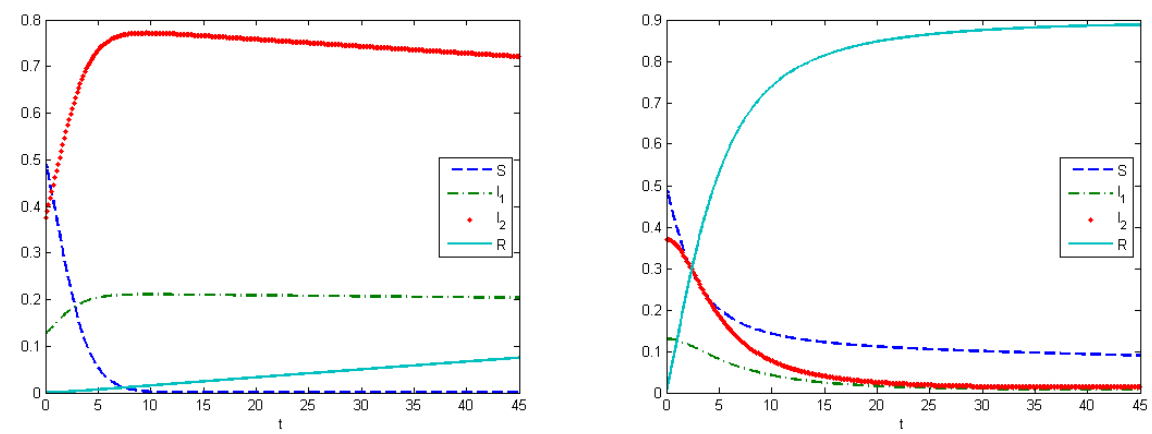

Fig. 5. Experiment 1.2. Left: SIIR model with virus mutation and without applying control. Maximum values are $I_{1 \max }=0.2165, I_{2 \max }=0.7746$. Epidemic peaks reached at the 11th and 8th days. Right: SIIR model with virus mutation and application of control. Functions $h_{i}\left(u_{i}(t)\right)$ are convex. Vertical axes show the fractions of the subpopulations. 

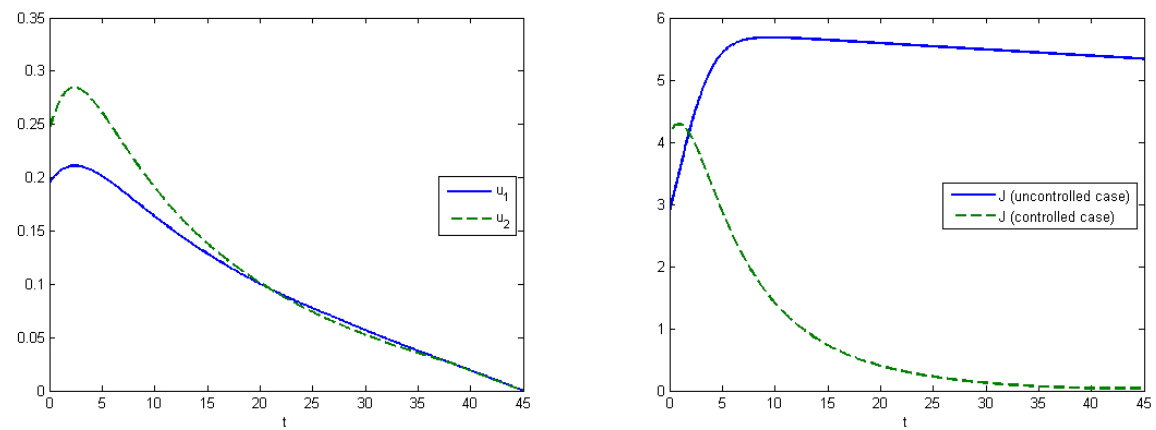

Fig. 6. Experiment 1.2. Left: Optimal control in the SIIR model with virus mutation and convex cost functions $h_{i}\left(u_{i}(t)\right)$. Vertical axis shows the amount of applied control. Right: Comparison of aggregated costs of the SIIR model with virus mutation (Controlled case: $J=73.64$, uncontrolled case: $J=261.4$ ). Vertical axis shows the aggregated costs at time moment $t$ in m.u.

Figure 5 (left) shows the behaviour of the system in the uncontrolled case, where the maximum population of $V_{1}$ was $I_{1}\left(t_{1}\right)=0.2165$ at $t_{1}=11.25$ and the maximum population of $V_{2}$ was $I_{2}\left(t_{2}\right)=0.7746$ at $t_{2}=8.5$. By applying optimal treatment strategies, we observed that the maximal values for both types of viruses were equal to their initial state $\left(I_{1}(0)=0.12\right.$ and $\left.I_{2}(0)=0.38\right)$. The structure of the optimal control in the case with the virus mutation is shown in Figure 6 (left). Comparison of aggregated costs is presented in Figure 6 (right). The aggregated system costs are $J=261.4$ in the uncontrolled case and $J=73.64$ in the controlled case. Figure 7 demonstrates the change of the infection rates of Experiment 1.2 in uncontrolled and controlled cases.
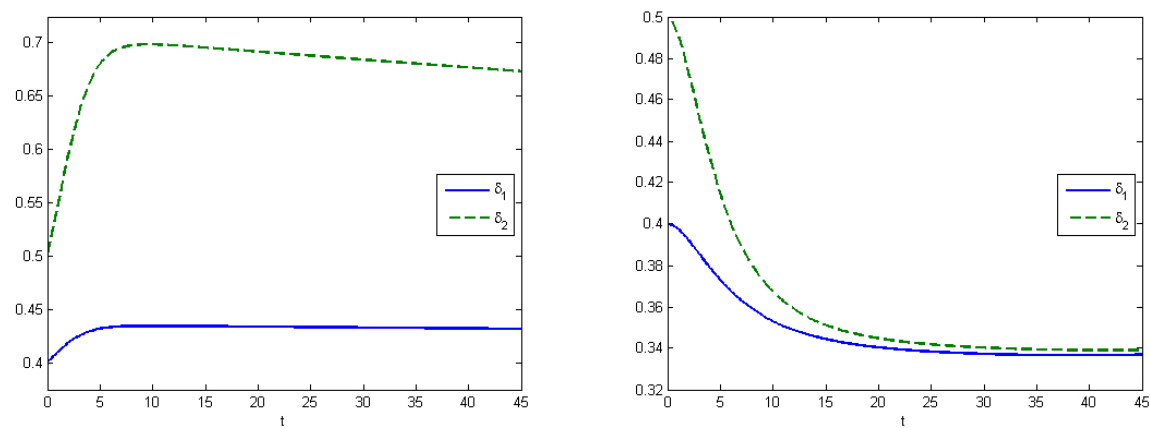

Fig. 7. Left: Experiment 1.3. Infected rates of the SIIR model with virus mutations (uncontrolled case). Infectious rates are $\delta_{1}=0.44$ and $\delta_{2}=0.67$ at the time moment $t=T$. Right: Experiment 3. Infected rates of the SIIR model with virus mutations (controlled case). Infectious rates are $\delta_{1}=0.337$ and $\delta_{2}=0.339$ at the time moment $t=T$.

Experiment 1.3 describes the case when the costs for medical treatments are concave functions. In this case, we have proven in Section 4.2. that the optimal control has a "bang-bang" structure, i.e., Figure 8 (right). The control for the first 
type of virus is turned off on the seventh day, and, for the second type, on the ninth day. At the end of the interval $T=45$, the proportion of the recovered hosts is $60 \%$, while the remaining population was still susceptible to infection. The comparisons of the aggregated costs are presented in Figure 9 (left). Aggregated system costs are $J=72.35$ in the uncontrolled case and $J=289.42$ in the controlled case.
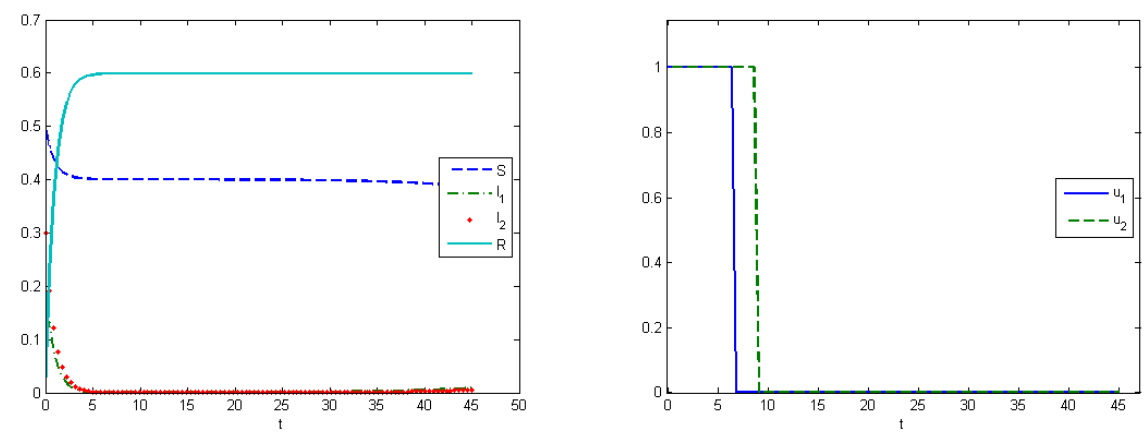

Fig. 8. Experiment 1.3. Left: SIIR model with virus mutation and application of control. Functions $h_{i}\left(u_{i}(t)\right)$ are concave. Vertical axis shows the fractions of the subpopulations. Right: Optimal control in SIIR model with virus mutation and concave-cost functions $h_{i}\left(u_{i}(t)\right)$. Control was switched off at the seventh day for $V_{1}$, and at the ninth day for $V_{2}$. Vertical axis shows the amount of applied control at time moment $t$.

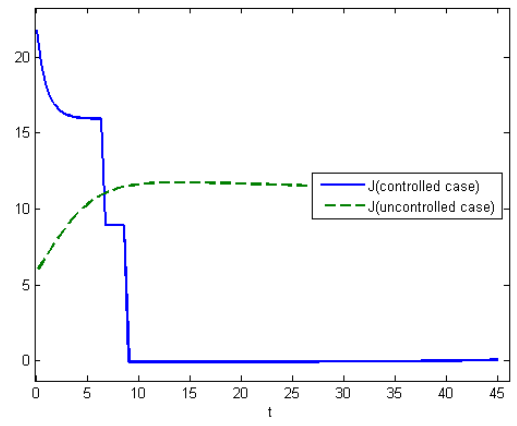

Fig. 9. Experiment 1.3. Comparison of aggregated costs of the SIIR model with virus mutation (Controlled case: $J=72.35$, uncontrolled case: $J=258.85$ ). Vertical axis shows the aggregated costs at time moment $t$ in m.u.

Experiment 1.4. The current simulations show the behaviour of the system on the time interval of 30 days. The next values of the parameters have been used in the experiments: infection rates are equal to $\delta_{1_{0}}=0.3$ and $\delta_{2_{0}}=0.4$, self-recovery rates are $\sigma_{1}=0.002$ and $\sigma_{2}=0.0012$ for virus $V_{1}$ and $V_{2}$ respectively. Uncontrolled and controlled epidemic processes are represented in Fig. 10. Medical treatment of the infected host is considered as a control strategy. Treatment costs function are in the convex form $h_{1}\left(u_{1}\right)=12 u_{1}^{2}$ and $h_{2}\left(u_{2}\right)=16 u_{2}^{2}$ and infection cost functions are $f_{1}\left(I_{1}(t)\right)=8 I_{1}(t), f_{2}\left(I_{2}(t)\right)=12 I_{2}(t)$. The structure of the optimal control and 
the aggregated system costs are presented in Fig. 11. Aggregated system costs are $J=268.79$ in the uncontrolled case and $J=59.2$ in the controlled case.
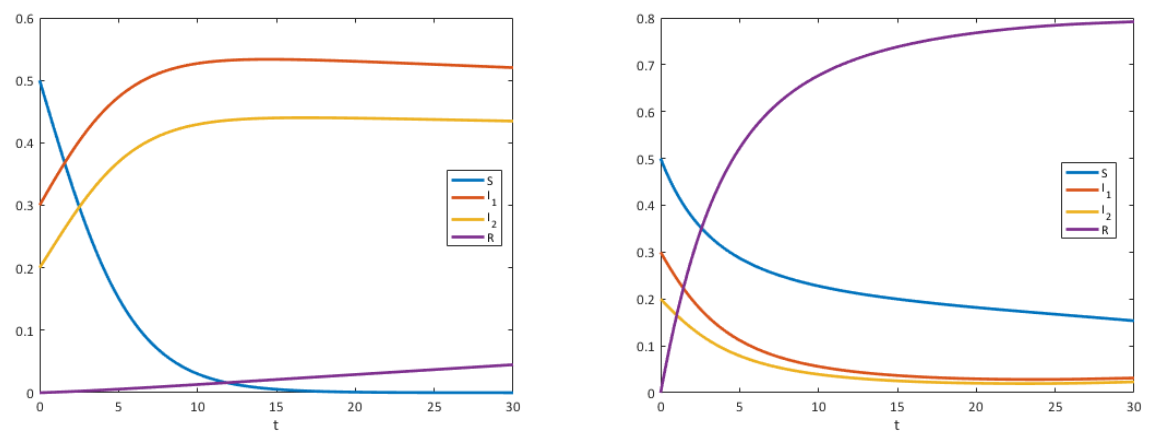

Fig. 10. Experiment 1.4. Left: SIIR model without virus mutation and without applying control. Initial states are $I_{1}(0)=0.3, I_{2}(0)=0.2$, maximum values are $I_{1 \max }=0.53$ and $I_{2 \max }=0.42$. Epidemic peaks a reached at 14 th and 15 th days. Right: SIIR model without virus mutation with application of control. Vertical axes show the fractions of the subpopulations.
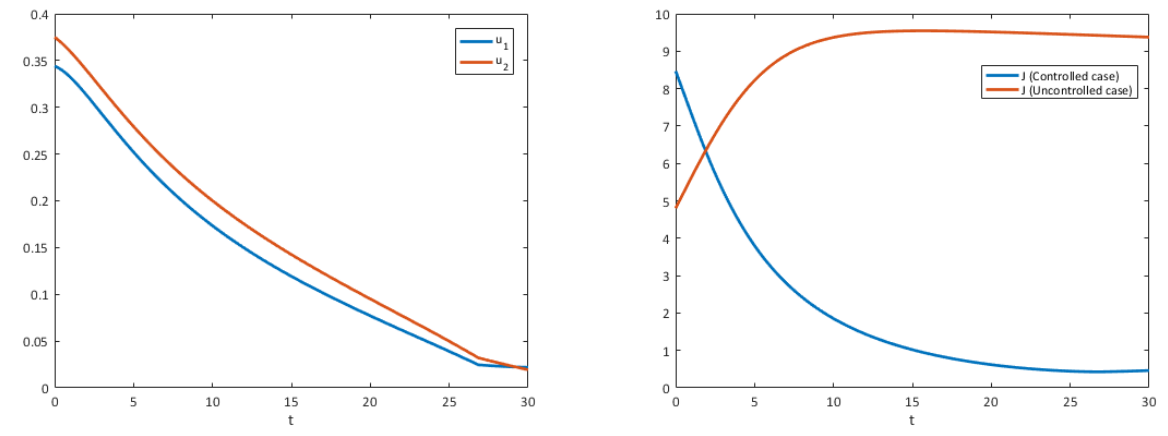

Fig. 11. Experiment 1.4. Left: Optimal control in SIIR model without virus mutation; cost functions $h_{i}\left(u_{i}\right)$ are convex. Vertical axis shows the amount of applied control. Right: Comparison of aggregated costs of SIIR model without virus mutation (Controlled case: $J=59.2$; uncontrolled case: $J=268.79$ ). Vertical axis shows the aggregated costs at time moment $t$ in m.u.

\section{SIIR-model over a complex network with continuous control}

Network frameworks are a natural modification of the epidemic model and provide a convenient tool for describing contacts between agents in a population. Such modification of the model from the Section 3. has been represented in (Taynitskiy, $2017 \mathrm{~b}$ ), where the spreading of the two different types of viruses is described over a complex network. In addition the network structure is the natural completion of the epidemic process because in many real-life situations, people can contact not only at random but in compliance with it is a network of relatives and neighbours. 
The same considerations can be applied to the computer and social networks. According to this reason we reformulate the epidemic model with two viruses in terms of networks.

Denote by $S_{k}(t), R_{k}(t)$ the population densities of the Susceptible and Recovered nodes with degree $k$ at time $t$. We consider two strains of viruses co-exist in the network. $I_{k}^{1}(t), I_{k}^{2}(t)$ are the population densities of the Infected nodes of degree $k$ at time $t$. In the network and for all $t$, we assume that the total population is constant, i.e., $S_{k}(t)+I_{k}^{1}(t)+I_{k}^{2}(t)+R_{k}(t)=1$ for any $k$. As in previous section, we have extended the simple SIR model (Kermack, 1927) to describe the propagation two different viruses over a population, which is defined by complex network.

$$
\begin{aligned}
\frac{d S_{k}}{d t} & =-\delta_{1} S I_{1} \Theta_{1}-\delta_{2} S I_{2} \Theta_{2} ; \\
\frac{d I_{k}^{1}}{d t} & =\left(\delta_{1} S_{k} \Theta_{1}-\sigma_{1}-u_{1}\right) I_{k}^{1} ; \\
\frac{d I_{k}^{2}}{d t} & =\left(\delta_{2} S_{k} \Theta_{2}-\sigma_{2}-u_{2}\right) I_{k}^{2} ; \\
\frac{d R_{k}}{d t} & =\left(\sigma_{1}+u_{1}\right) I_{k}^{1}+\left(\sigma_{2}+u_{2}\right) I_{k}^{2} ;
\end{aligned}
$$

where $\delta_{i}$ are infection rates for virus $V_{i}, i=1,2, \sigma_{i}$ are recovered rates, and let infectivity of the be denoted as $l_{i}=\delta_{i} / \sigma_{i}$.

We considered the graph generated by using the algorithm devised in (Barabasi, 1999). We start from a small number $m_{0}$ of disconnected nodes; every time step a new node is added, with $m$ links that are connected to an old node $i$ with $k_{i}$ links according to the probability $k_{i} / \sum_{j} k_{j}$. After iterating this scheme a sufficient number of times, we obtain a network composed by $N$ nodes with connectivity distribution $P(k) \approx k^{-3}$ and average connectivity $\langle k\rangle=2 m$. In this work, we take $m=4$.

At the beginning of epidemic process $t=0$, most of nodes in the network belong to the susceptible subgroup, and small subgroup in total population is infected; and the remaining nodes are in the recovered subgroup. Hence initial states are: $0<S_{k}(0)=S^{0}<1,0<I_{k}^{1}(0)<1,0<I_{k}^{2}(0)<1, R_{k}(0)=1-S^{0}-I_{k}^{1}(0)-I_{k}^{2}(0)$. $\Theta_{i}(t)$ can be written in general (see (Fu, 2008; Vespignani, 2015)) as

$$
\Theta_{i}\left(l_{i}\right)=\sum_{k^{\prime}} \frac{l_{i} P\left(k^{\prime} \mid k\right) I_{k^{\prime}}^{j}}{k^{\prime}}, i=1,2,
$$

where $P\left(k^{\prime} \mid k\right)$ describes the probability of a node with degree $k$ pointing to a node with degree $k^{\prime}$, and $P\left(k^{\prime} \mid k\right)=\frac{k^{\prime} P\left(k^{\prime}\right)}{\langle k\rangle}$, where $\langle k\rangle=\sum_{k^{\prime}} k P(k)$. For scale-free node distribution $P(k)=C^{-1} k^{-2-\gamma}, 0<\gamma \leq 1$, where $C=\zeta(2+\gamma)$ is Riemann's zeta function, which provides the appropriate normalization constant for sufficiently large networks. The control parameters which can be used to protect the network from the propagation of the virus are defined as $u=\left(u_{1}, u_{2}\right)$. Here, $u_{i}$ are fractions of the infected nodes which are quarantined in the population. Recovered rates $\sigma_{i}$ are the coefficients of "self-recovery", which defined the self-recovery process, without application of any special measures.

\subsection{Objective Function}

As in previous section, we will minimize the overall cost in time interval $[0, T]$. At any given $t$, the following cost functions are presented in the system: $f_{1}\left(I_{k}^{1}(t)\right)$, 
$f_{2}\left(I_{k}^{2}(t)\right)$ are infection costs; $g\left(R_{k}(t)\right)$ is utility of having $R_{k}(t)$ fraction of nodes recovered at time $t ; h_{1}\left(u_{1}(t)\right), h_{2}\left(u_{2}(t)\right)$ are costs for using antivirus patches or quarantine that help to reduce epidemic spreading. Here, functions $f_{i}(t(t))$ are nondecreasing and twice-differentiable, convex functions, with $f_{i}(0)=0, f_{i}\left(I_{i}(t)\right)>0$ for $I_{i}(t)>0, i=1,2 ; g(\cdot)$ is non-decreasing and differentiable, and $g(0)=0$; $h_{i}\left(u_{i}(t)\right)$ is twice-differentiable and increasing function in $u_{i}(t)$ such that $h_{i}(0)=0$, $h_{i}\left(u_{i}(t)\right)>0, i=1,2$ when $u_{i}(t)>0$.

The aggregated system costs is given by

$$
J=\int_{0}^{T} f_{1}\left(I_{k}^{1}(t)\right)+f_{2}\left(I_{k}^{2}(t)\right)-g\left(R_{k}(t)\right)+h_{1}\left(u_{1}(t)\right)+h_{2}\left(u_{2}(t)\right) d t
$$

and the optimal control problem is to minimize the cost, i.e., $\min _{\left\{u_{1}, u_{2}\right\}} J$.

Treatment or isolation is considered as the control parameters that can help to reduce the fraction of infected nodes in network. We define variable $u=\left(u_{1}, u_{2}\right)$ as control variables with $0 \leq u_{1}(t) \leq 1,0 \leq u_{2}(t) \leq 1$, for all $t$.

\subsection{Optimal control of SIIR-model over a complex network with continuous control}

As previously, we find the optimal solution $u=\left(u_{1}, u_{2}\right)$ by using Pontryagin's maximum principle (Pontryagin, 1987).

Since the algorithm of finding the optimal control repeats steps from the Section 1, we represent auxiliary computation in Appendix 1, and in the current paragraph, only the structure of the optimal control is shown. Firstly, switching functions $\varphi_{k}^{i}$ are defined as follows:

$$
\varphi_{k}^{1}=\left(\lambda_{I_{k}^{1}}-\lambda_{R_{k}}\right) I_{k}^{1}, \quad \varphi_{k}^{2}=\left(\lambda_{I_{k}^{2}}-\lambda_{R_{k}}\right) I_{k}^{2} .
$$

Proposition 1. If $h(\cdot)$ and the Hamiltonian are concave, then the optimal control policy has following structure:

$$
u_{i}^{*}(t)= \begin{cases}0, & \text { for } \varphi_{k}^{i}(t)<h_{i}(1) \\ 1, & \text { for } \varphi_{k}^{i}(t) \geq h_{i}(1)\end{cases}
$$

If $h(\cdot)$ and the Hamiltonian are convex, then the optimal control policy has following structure:

$$
u_{i}^{*}(t)= \begin{cases}0, & \text { for } \varphi_{k}^{i}(t) \leq h_{i}^{\prime}(0) \\ h^{\prime-1}\left(\varphi_{k}^{i}\right), & \text { for } h_{i}^{\prime}(0)<\varphi_{k}^{i}(t) \leq h_{i}^{\prime}(1) \\ 1, & \text { for } h_{i}^{\prime}(1)<\varphi_{k}^{i}(t) .\end{cases}
$$

\section{SIIS-model over a complex network with continuous control}

A set of nodes $N$ is divided into two subgroups: Susceptible $(S)$, Infected $(I)$. We suppose that two different viruses with different strains circulate in the network at time $t$. Let $S_{k}(t), I_{k}^{1}(t), I_{k}^{2}(t)$ be the densities of susceptible and infected nodes with degree $k$ at time $t$. Let $l_{i}=\frac{\delta_{i}}{\sigma_{i}}$, where $\delta_{i}$ is infection rate and infected nodes are cured and become again susceptible with rate $\sigma_{i}, i=1,2$. 


$$
\begin{aligned}
\frac{d S_{k}}{d t} & =-l_{1} S_{k}(t) \Theta_{1}-l_{2} S_{k}(t) \Theta_{2}+\left(u_{1}+1\right) I_{k}^{1}(t)+\left(u_{2}+1\right) I_{k}^{2}(t) ; \\
\frac{d I_{1}}{d t} & =l_{1} S_{k}(t) \Theta_{1}-I_{k}^{1}(t)-u_{1} I_{k}^{1}(t) \\
\frac{d I_{2}}{d t} & =l_{2} S_{k}(t) \Theta_{2}-I_{k}^{2}(t)-u_{2} I_{k}^{2}(t) .
\end{aligned}
$$

\subsection{Objective Function}

At any given $t$ in time interval $[0, T]$ following costs exist in the system: $f\left(I_{k}(t)\right)$ these are treatment costs, $h_{i}\left(u_{i}(t)\right)$ are costs for medical measures (i.e. quarantine) that help to reduce epidemic spreading. Here, the functions $f_{i}(\cdot)$ are non-decreasing and twice-differentiable, convex functions, with $f_{i}(0)=0, f_{i}\left(I_{k}^{i}\right)>0$ for $I_{k}^{i}>0$, $g\left(S_{k}(t)\right)$ is non-decreasing and differentiable function describing benefits of using control, where $S_{k}(t)=1-I_{k}^{1}(t)-I_{k}^{2}(t)$ and $g(0)=0 ; h_{i}(\cdot)$ are twice-differentiable and increasing function in $u_{i}(t)$ such that $h_{i}(0)=0, h_{i}\left(u_{i}\right)>0$ when $u_{i}>0$, with feasible controls $u_{i} \in[0,1]$.

The aggregated system costs is given by

$$
J=\int_{0}^{T} f_{1}\left(I_{k}^{1}(t)\right)+f_{2}\left(I_{k}^{2}(t)\right)+h_{1}\left(u_{1}(t)\right)+h_{2}\left(u_{2}(t)\right)-g\left(S_{k}(t)\right) d t
$$

and the optimal control problem is to minimize the cost, i.e., $\min _{u_{1}, u_{2} \in[0,1]} J$. System (17) describes the propagation of two different strains of viruses in the network. The propagation of the viruses is controlled by parameters $u_{i}, i=1,2$. Here $u_{i}$ is the antivirus strategy.

\subsection{Optimal control of SIIS-model over a complex network with continuous control}

We find the optimal control $u=\left(u_{1}, u_{2}\right)$ which yields the minimum solution to the functional (18) for the problem described above following Pontryagin's maximum principle. The Hamiltonian of the system and the adjoint functions can be found in Appendix 2. Define switching functions $\varphi_{k}^{i}$ as follows:

$$
\varphi_{k}^{1}=\left(\lambda_{I_{k}^{1}}-\lambda_{S_{k}}\right) I_{k}^{1} ; \quad \varphi_{k}^{2}=\left(\lambda_{I_{k}^{2}}-\lambda_{S_{k}}\right) I_{k}^{2} .
$$

Proposition 2. If $h(\cdot)$ are concave, then the optimal control policy has following structure:

$$
u_{i}^{*}(t)= \begin{cases}0, & \text { for } \varphi_{k}^{i}(t)<h_{i}(1) \\ 1, & \text { for } \varphi_{k}^{i}(t) \geq h_{i}(1)\end{cases}
$$

If $h(\cdot)$ is convex, then the optimal control policy has following structure:

$$
u_{i}^{*}(t)= \begin{cases}0, & \text { for } \varphi_{k}^{i}(t) \leq h_{i}^{\prime}(0) \\ h^{\prime-1}\left(\varphi_{k}^{i}\right), & \text { for } h_{i}^{\prime}(0)<\varphi_{k}^{i}(t) \leq h_{i}^{\prime}(1) ; \\ 1, & \text { for } h_{i}^{\prime}(1)<\varphi_{k}^{i}(t) .\end{cases}
$$

\section{Numerical simulation of SIIR and SIIS models with continuous control}

In this section, series of numerical simulations are represented in support of the main theoretical results from the Section 6. We represent optimal strategies for 
SIIR and SIIS models for strictly convex cost functions $h_{i}\left(u_{i}\right)$ and compare the aggregated system costs in uncontrolled and controlled cases.

Experiment 2.1. This experiment represents the behavior of the SIIR system. We use the following initial states: susceptible subpopulation $-S_{k}(0)=0.5$, infected - $I_{k}^{1}(0)=0.2$ and $I_{k}^{2}(0)=0.3$, and recovered $-R_{k}(0)=0$. Infection rates are $\delta_{1}=0.3$ and $\delta_{2}=0.4$ for the first and the second viruses, respectively. Self-recovery rates are $\sigma_{1}=0.003$ and $\sigma_{2}=0.001$. We consider time interval $[0, T]$, where $T=$ 20. Infected cost functions incurred by the virus are $f_{I_{k}^{1}(t)}=8 I_{k}^{1}(t)$ and $f_{I_{k}^{2}(t)}=$ $10 I_{k}^{2}(t)$. Utility function from the fraction of the recovered nodes $R_{k}(t)$ are equal to $g\left(R_{k}(t)\right)=0.1 R_{k}(t)$. Treatment cost functions are strictly convex $-h_{1}\left(u_{1}(t)\right)=$ $0.4 u_{1}^{2}(t)$ for the first virus and $h_{2}\left(u_{2}(t)\right)=0.5 u_{2}^{2}(t)$ for the second virus.
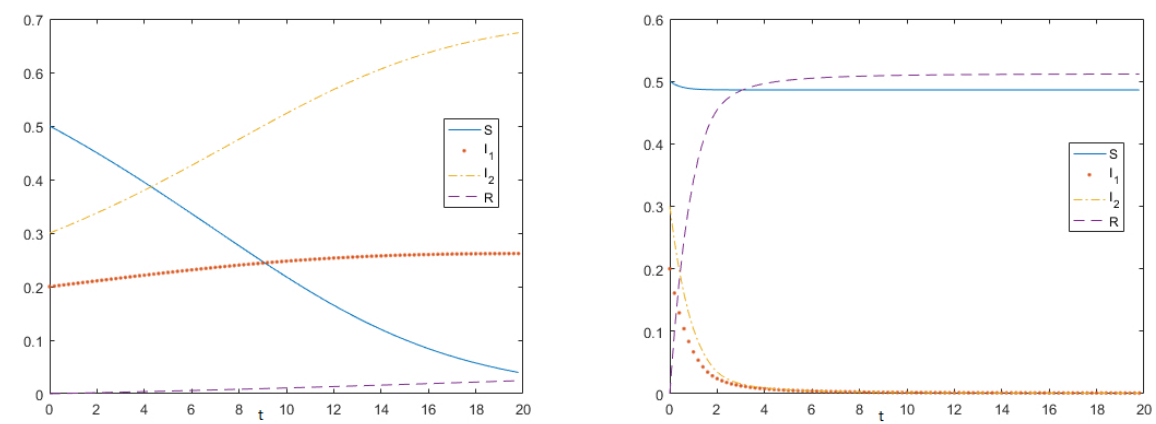

Fig. 12. Experiment 2.1. Left: SIIR model without applying of control. Right: SIIR with application of control.

Behavior of the system (12) is presented in Figure 12: left - uncontrolled case, right - controlled case. In uncontrolled case, most of the nodes are infected by one of the viruses and a small fraction of the nodes are recovered. Epidemic peaks a reached at the last 20-th day. In controlled case, at the end of the interval, all nodes are in the recovered or susceptible states.
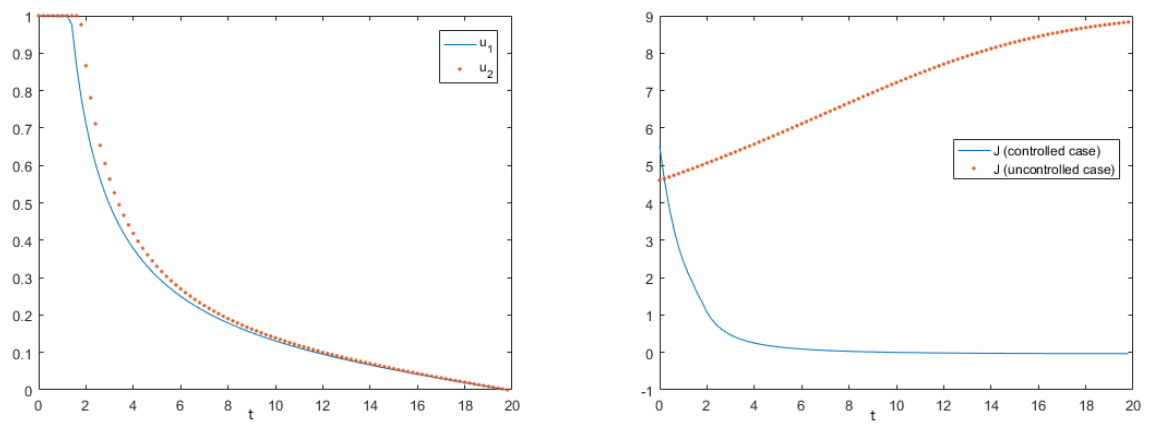

Fig. 13. Experiment 2.1. Left: Structure of the optimal control in SIIR model. Right: Comparison of aggregated costs of SIIR model without virus mutation (Controlled case: $J=36.39$; uncontrolled case: $J=175.25$ ). 
The optimal control strategy is shown in Figure 13(left). The set of switching points are $t_{1}=1.4$ and $t_{2}=1.8$. Aggregation system costs are equal to $J_{0}=175.25$ when treatment is not applied to the infected group, and $J_{1}=36.39$ in controlled case (Figure 13(right)).

Experiment 2.2. For the case of SIIS model, we use the following initial state values: $S_{k}(0)=0.7, I_{k}^{1}(0)=0.1, I_{k}^{2}(0)=0.2$; infection and recovered rates are the same as in Experiment 2.1. The total duration of the epidemics in this experiment remains the same $T=20$. We also keep the values of all cost functions. Behavior of the system (17) in uncontrolled and controlled cases are presented in Figure 14.
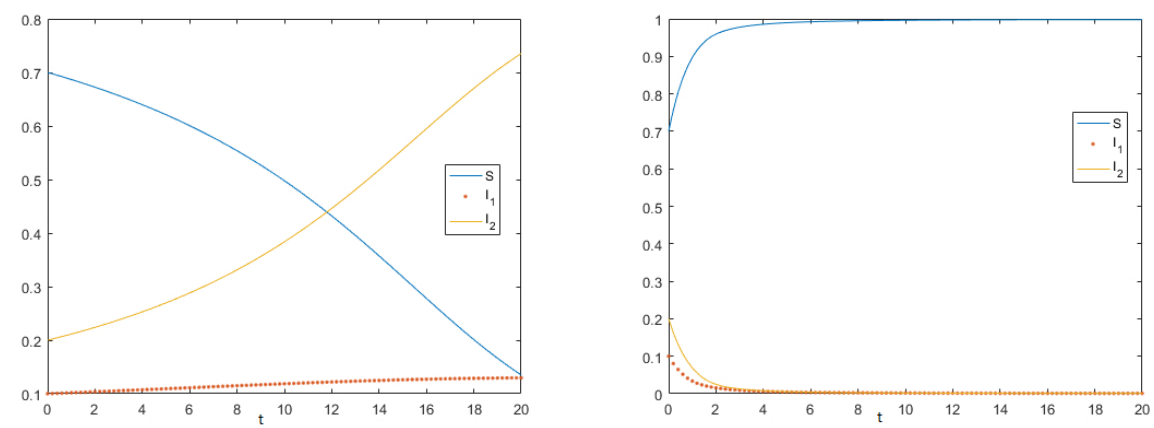

Fig. 14. Experiment 2.2. Left: SIIS model in uncontrolled case. Initial states are $I_{1}(0)=$ $0.1, I_{2}(0)=0.2$, the maximum values are $I_{1 \max }=0.12, I_{2 \max }=0.73$. Epidemic peaks a reached at the last 20-th day. Right: SIIS with application of control.

The optimal control strategy is shown in Figure 15(left). Aggregation system costs are $J_{0}=124.6$ when in uncontrolled case, and $J_{1}=19.93$ in controlled case (Figure 15(left)).
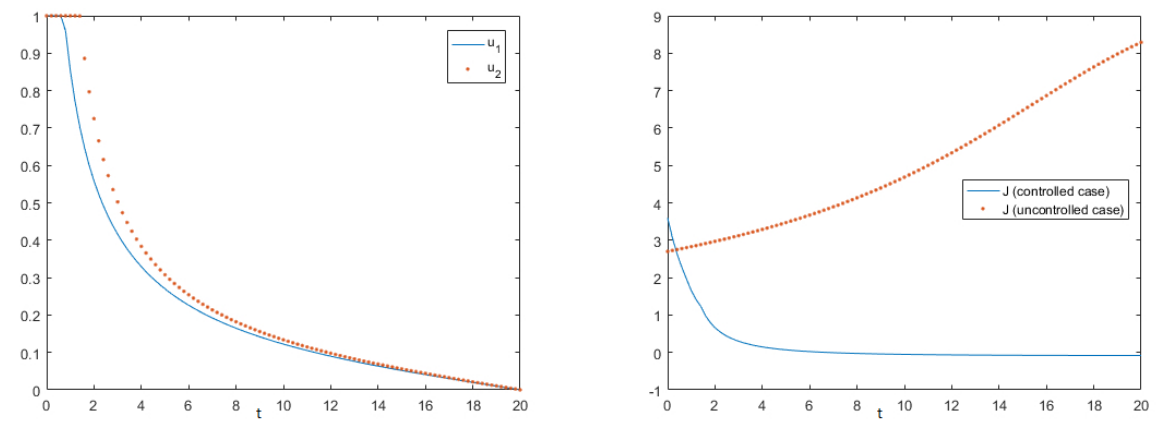

Fig. 15. Experiment 2.2. Left: Optimal control in SIIS model without virus mutation, costs functions are convex $h_{i}\left(u_{i}\right)$. Switching points are $t_{1}=0.8$ and $t_{2}=1.6$. Right: Comparison of aggregated costs of SIIS model without virus mutation (Controlled case: $J=19.93$, uncontrolled case: $J=124.6$ ). 


\section{SIIR-model over a complex network with impulse control}

In the current section, we consider a model of spreading of viruses in the network of $N$ nodes, based on the modification of classical SIR model from sections 3. and 6 . Following the previous works (Gubar, 2013; Taynitskiy, 2016b), two different forms of viruses with different strengths spread over the network simultaneously, we denote them as $V_{1}$ and $V_{2}$. It is also assumed that that a structure of population is described by the scale free network (Vespignani, 2015; Gubar, 2015). Normally, as SIR model points, all nodes in the population are divided into three groups: Susceptible $(S)$, Infected $(I)$ and Recovered $(R)$. Susceptible is a group of nodes which are not infected by any virus, but may be invaded by any form of virus. The Infected nodes are those that have been attacked by the virus and the Recovered is a group of recovered nodes. In modified model subgroup of Infected nodes also is brunched into two subgroups $I_{1}$ and $I_{2}$, where nodes in $I_{i}$ are infected by virus $V_{i}, i=1,2$. We formulate the epidemic process as a system of nonlinear differential equations, where $n_{S}$, $n_{V_{1}}, n_{V_{2}}$ and $n_{R}$ correspond to the number of susceptible, infected and recovered nodes, respectively. In current model the connections between nodes are described by the scale-free network, then we will use the following notation: $S_{k}(t)$ and $R_{k}(t)$ are fractions of Susceptible and Recovered nodes with degree $k$ at time moment $t, I_{k}^{1}(t), I_{k}^{2}(t)$ are fractions of Infected nodes with degree $k$. At each time moment $t \in[0, T]$ the number of nodes is constant and equal $N$, and the following condition $S_{k}(t)+I_{k}^{1}(t)+I_{k}^{2}(t)+R_{k}(t)=1$ is satisfied for any $k$. The process of spreading is defined by the system of ordinary differential equations:

$$
\begin{aligned}
\frac{d S_{k}}{d t} & =-\delta_{1 k} S_{k} I_{k}^{1} \Theta_{1}-\delta_{2 k} S_{k} I_{k}^{2} \Theta_{2} \\
\frac{d I_{k}^{1}}{d t} & =\delta_{1 k} S_{k} I_{k}^{1} \Theta_{1}-\sigma_{k}^{1} I_{k}^{1} \\
\frac{d I_{k}^{2}}{d t} & =\delta_{2 k} S_{k} I_{k}^{2} \Theta_{2}-\sigma_{k}^{2} I_{k}^{2} \\
\frac{d R_{k}}{d t} & =\sigma_{k}^{1} I_{k}^{1}+\sigma_{k}^{2} I_{k}^{2}
\end{aligned}
$$

where $\delta_{i k}(k)$ is the infections rate for the first type of the virus $i$ if a susceptible node has a contact with infected node with the degree $k, \sigma_{k}^{i}$ is recovery rate.

At the initial time moment $t=0$, the most number of nodes belong to Susceptible group and only a small fraction of Infected by viruses $V_{1}$ or $V_{2}$. Initial state for system $(22)$ is $0<S_{k}(0)<1,0<I_{k}^{1}(0)<1,0<I_{k}^{2}(0)<1, R_{k}(0)=1-S_{k}(0)-$ $I_{k}^{1}(0)-I_{k}^{2}(0)$. Analogously with (Fu, 2008; Vespignani, 2015) we define parameter $\Theta_{i}(t)$ as

$$
\Theta_{i}\left(l_{i}\right)=\sum_{k^{\prime}} \frac{\delta_{i k} P\left(k^{\prime} \mid k\right) I_{k^{\prime}}^{i}}{k^{\prime}}, i=1,2 .
$$

\subsection{Impulse control problem}

Previously it was shown in (Vespignani, 2015) a small fraction of the infected nodes might be survived on small segments of the network and can provoke new waves of epidemics. This cycled process recalls the behavior of the virus of influenza which causes a seasonally periodic epidemic, (Agur, 1993). Nowadays, several respiratory viruses, including SARS-COV-2 are able to cause repeated waves of the disease. Hence the control of the epidemic process can be formulated as an impulse 
control problem in which a series of impulses of antivirus patches are designed to reduce the periodically incipient zones of infected nodes. We extend the model (22) to present an impulse control problem for resuming of virus attacks and obtain the optimal strategies, which prevent the spreading of viruses in discrete time moments.

We suppose that impulses occur at time $\tau_{k, 1}^{i}, \ldots, \tau_{k, q_{i}(k)}^{i}$, where $q_{i}(k)$ describes the number of launching of impulse controls for nodes with $k$ degrees, index $i$ indicates the type of virus. We also assume that on the time intervals $\left(\tau_{k, j}^{i}, \tau_{k, j+1}^{i}\right]$ system (22) describes the behaviour of virus in the network. We have reformulated epidemic model to describe the situation with two types of viruses for all time periods except the sequence of times $\tau_{k, j}^{i+}, j=1, \ldots, q_{i}(k), i=1,2$. Additionally, we set $S\left(\tau_{k, j}^{i}\right)=S\left(\tau_{k, j}^{i-}\right), I_{1}\left(\tau_{k, j}^{i}\right)=I_{1}\left(\tau_{k, j}^{i-}\right), I_{2}\left(\tau_{k, j}^{i}\right)=I_{2}\left(\tau_{k, j}^{i-}\right), R\left(\tau_{k, j}^{i}\right)=R\left(\tau_{k, j}^{i-}\right)$.

The system after activation of impulses at time moment $\tau_{k, j}^{i+}$ is:

$$
\begin{aligned}
& S_{k}\left(\tau_{k, j}^{i+}\right)=S_{k}\left(\tau_{k, j}^{i}\right), \\
& I_{k}^{1}\left(\tau_{k, j}^{i+}\right)=I_{k}^{1}\left(\tau_{k, j}^{i}\right)-\nu_{k}^{1}\left(\tau_{k, j}^{i}\right), \\
& I_{k}^{2}\left(\tau_{k, j}^{i+}\right)=I_{k}^{2}\left(\tau_{k, j}^{i}\right)-\nu_{k}^{2}\left(\tau_{k, j}^{i}\right), \\
& R_{k}\left(\tau_{k, j}^{i+}\right)=R_{k}\left(\tau_{k, j}^{i}\right)+\nu_{k}^{1}\left(\tau_{k, j}^{i}\right)+\nu_{k}^{2}\left(\tau_{k, j}^{i}\right) .
\end{aligned}
$$

Variables $\nu_{k}^{i}=\left(\nu_{k, 1}^{i}, \ldots, \nu_{k, q_{i}(k)}^{i}\right), i=1,2$, correspond to control impulses applied at the discrete time moments $\tau_{k, 1}, \ldots, \tau_{k, q_{i}(k)}$ and represent the fraction of recovered nodes. Let be $\nu_{k, j}^{i}=c_{k, j}^{i} \delta\left(t-\tau_{k, j}^{i}\right)$, where $\delta\left(t-\tau_{k, j}^{i}\right)$ is Dirac function, $c_{k, j}^{i} \in\left[0, \bar{u}_{k, j}^{i}\right]$ is the value of impulse, leads to changes of the dynamical system, $\bar{u}_{k, j}^{i}$ is the maximum value for control (Agur, 1993).

\subsection{Objective Function}

The objective function of the combined system (24) is represented by the aggregated costs on the time interval $[0, T]$ including the costs of control impulses. The aggregated costs for continuous system (22) are defined as follows: at time moment $t \neq \tau_{k, j}^{i}, j=1, \ldots, q_{i}(k), i=1,2$, we have the costs from infected nodes $f_{k}^{1}\left(I_{k}^{1}(t)\right)$ and $f_{k}^{2}\left(I_{k}^{2}(t)\right)$. Functions $f_{k}^{i}(\cdot)$ are non-decreasing and twice-differentiable, such that $f_{k}^{i}(0)=0, f_{k}^{i}\left(I_{k}^{i}(t)\right)>0$ for $I_{k}^{i}(t)>0$ with $t \in\left(\tau_{k, j-1}^{i}, \tau_{k, j}^{i}\right]$. For system (24), we define the treatment costs as functions $h_{k}^{i}\left(\nu_{k, j}^{i}\left(\tau_{k, j}^{i+}\right)\right), j=1, \ldots, q_{i}(k)$, where $h_{k}^{i}\left(\nu_{k, j}^{i}\left(\tau_{k, j}^{i+}\right)\right)>0, \nu_{k, j}^{i}\left(\tau_{k, j}^{i+}\right)>0$ for $i=1,2$. Functions $g\left(R_{k}(t)\right)$ are nondecreasing and capture the benefit rates from Recovered nodes. The aggregated system costs are defined by the functional:

$$
\begin{array}{r}
J=\sum_{k \in \mathbb{N}}\left[\int_{0}^{T} f_{k}^{1}\left(I_{k}^{1}(t)\right)+f_{k}^{2}\left(I_{k}^{2}(t)\right)-g\left(R_{k}(t)\right) d t+\sum_{j=1}^{q_{1}(k)} h_{k}^{1}\left(\nu_{k, j}^{1}\left(\tau_{k, j}^{1}\right)\right)+\right. \\
\left.\sum_{j=1}^{q_{2}(k)} h_{k}^{2}\left(\nu_{k, j}^{2}\left(\tau_{k, j}^{2}\right)\right)\right] .
\end{array}
$$

\subsection{The structure of impulse control}

According to principle maximum in impulse form (Blaquiere, 1985; Chahim, 2012; Dykhta, 2009; Taynitskiy, 2015) we write Hamiltonian for dynamic system (22)

$$
\begin{aligned}
H_{k}^{0}(t)= & -f_{k}^{1}\left(I_{k}^{1}(t)\right)-f_{k}^{2}\left(I_{k}^{2}(t)\right)+g\left(R_{k}(t)\right)+\left(\lambda_{I_{k}^{1}}(t)-\lambda_{S_{k}}(t)\right) \delta_{1 k} S_{k}(t) I_{k}^{1}(t) \Theta_{1}(t)+ \\
& \left(\lambda_{I_{k}^{2}}(t)-\lambda_{S_{k}}(t)\right) \delta_{2 k} S_{k}(t) I_{k}^{2}(t) \Theta_{2}(t)+\left(\lambda_{R_{k}}-\lambda_{I_{k}^{1}}\right) \sigma_{k}^{1} I_{k}^{1}+\left(\lambda_{R_{k}}-\lambda_{I_{k}^{2}}\right) \sigma_{k}^{2} I_{k}^{2} ;
\end{aligned}
$$


and construct adjoint system as follows:

$$
\begin{aligned}
\dot{\lambda}_{S_{k}}(t)= & \left(\lambda_{S_{k}}(t)-\lambda_{I_{k}^{1}}(t)\right) \delta_{1 k} I_{k}^{1}(t) \Theta_{1}(t)+\left(\lambda_{S_{k}}(t)-\lambda_{I_{k}^{2}}(t)\right) \delta_{2 k} I_{k}^{2}(t) \Theta_{2}(t) ; \\
\dot{\lambda}_{I_{k}^{1}}(t)= & \frac{d f_{k}^{1}\left(I_{k}^{1}(t)\right)}{d I_{k}^{1}}+\left(\lambda_{S_{k}}(t)-\lambda_{I_{k}^{1}}(t)\right)\left(\delta_{1 k} S_{k}(t) \Theta_{1}(t)+\frac{\left(\delta_{1 k}\right)^{2} S_{k}(t) I_{k}^{1}(t) P(k)}{\langle k\rangle}\right)+ \\
& \left(\lambda_{I_{k}^{1}}-\lambda_{R_{k}}\right) \sigma_{k}^{1} ; \\
\dot{\lambda}_{I_{k}^{2}}(t)= & \frac{d f_{k}^{2}\left(I_{k}^{2}(t)\right)}{d I_{k}^{2}}+\left(\lambda_{S_{k}}(t)-\lambda_{I_{k}^{2}}(t)\right)\left(\delta_{2 k} S_{k}(t) \Theta_{2}(t)+\frac{\left(\delta_{2 k}\right)^{2} S_{k}(t) I_{k}^{2}(t) P(k)}{\langle k\rangle}\right)+ \\
& \left(\lambda_{I_{k}^{2}}-\lambda_{R_{k}}\right) \sigma_{k}^{2} ; \\
\dot{\lambda}_{R_{k}}(t)= & -\frac{d g\left(R_{k}(t)\right.}{d R_{k}},
\end{aligned}
$$

with transversality conditions $\lambda_{S_{k}}(T)=\lambda_{I_{k}^{1}}(T)=\lambda_{I_{k}^{2}}(T)=\lambda_{R_{k}}(T)=0$.

Following the maximum principle for impulse control (see Blaquiere, 1985; Sethi, 2006; Chahim, 2012), we formulate necessary optimality conditions as in Theorem 1.

Theorem 1. Let $\left(x^{*}, N, \tau_{i}^{j *}, \nu_{i}^{*}\right), i=1,2$, be an optimal solution for the impulse control problem. Then, there exists an adjoint vector function $\lambda(t)=\left(\lambda_{S}(t), \lambda_{I_{1}}(t)\right.$, $\left.\lambda_{I_{2}}(t), \lambda_{R}(t)\right)$ such that the following conditions hold:

$$
\dot{\lambda}_{x}(t)=-\frac{\partial H_{0}}{\partial x}\left(x^{*}(t), \lambda(t), t\right),
$$

where $x(t)=S(t), I_{1}(t), I_{2}(t), R(t)$.

At the impulse or jump points, it holds that

$$
\begin{gathered}
\frac{\partial H_{i}^{c}}{\partial \nu_{i}}\left(x^{*}\left(\tau_{i}^{j *-}\right), \nu_{i}, \lambda\left(\tau_{i}^{j *+}\right), \tau_{i}^{j *}\right)\left(\nu_{i}^{j}-\nu_{i}^{j *}\right) \geq 0, \\
\lambda_{x}\left(\tau_{i}^{j *+}\right)-\lambda_{x}\left(\tau_{i}^{j *-}\right)=\frac{\partial H_{i}^{c}}{\partial x}\left(x^{*}\left(\tau_{i}^{j *-}\right), \nu_{i}^{j *}, \lambda\left(\tau_{i}^{j *+}\right), \tau_{i}^{j *}\right), \\
H_{0}\left(x^{*}\left(\tau_{i}^{j *+}\right), \lambda\left(\tau_{i}^{j *+}\right), \tau_{i}^{j *}\right)-H_{0}\left(x^{*}\left(\tau_{i}^{j *-}\right), \lambda\left(\tau_{i}^{j *-}\right), \tau_{i}^{j *}\right)- \\
-\frac{\partial H_{i}^{c}}{\partial \tau_{i}^{j}}\left(x^{*}\left(\tau_{i}^{j *-}\right), \nu_{i}^{j *}, \lambda\left(\tau_{i}^{j *+}\right), \tau_{i}^{j *}\right)
\end{gathered}
$$

Equation (31) is strictly more than zero for $\tau_{i}^{j *}=0$, equal to zero for $\tau_{i}^{j *} \in$ $(0, T)$, and strictly less than zero for $\tau_{i}^{j *}=T$.

For all points in time at which there is no jump, i.e. $t \neq \tau_{j}\left(j=1, \ldots, k_{i}\right)$, it holds that

$$
\frac{\partial H_{j}^{c}}{\partial \nu_{j}}\left(x^{*}(t), 0, \lambda(t), t\right) \nu_{j} \leq 0,
$$

with the transversality condition $\lambda(T)=0$.

Hamiltonian in impulsive form is

$$
\begin{aligned}
& H_{k}^{c}\left(\tau_{k, j}^{1+}\right)=-h_{k}^{1}\left(\nu_{k, j}^{1}\left(\tau_{k, j}^{1+}\right)\right)+\left(\lambda_{R_{k}}\left(\tau_{k, j}^{1+}\right)-\lambda_{I_{k}^{1}}\left(\tau_{k, j}^{1+}\right)\right) \nu_{k, j}^{1}\left(\tau_{k, j}^{1+}\right) \\
& H_{k}^{c}\left(\tau_{k, j}^{2+}\right)=-h_{k}^{2}\left(\nu_{k, j}^{2}\left(\tau_{k, j}^{2+}\right)\right)+\left(\lambda_{R_{k}}\left(\tau_{k, j}^{2+}\right)-\lambda_{I_{k}^{2}}\left(\tau_{k, j}^{2+}\right)\right) \nu_{k, j}^{2}\left(\tau_{k, j}^{2+}\right) .
\end{aligned}
$$

Here we assume that for each type of virus $V_{1}$ and $V_{2}$ and for each $k$ we have own set of control impulses $\nu_{k}^{1}=\left(\nu_{k, 1}^{1}, \ldots, \nu_{k, q_{1}(k)}^{1}\right)$ and $\nu_{k}^{2}=\left(\nu_{k, 1}^{2}, \ldots, \nu_{k, q_{2}(k)}^{2}\right)$. 
Adjoin system for $\operatorname{system}(24)$ is $(i=1,2)$ :

$$
\begin{aligned}
& \lambda_{S_{k}}\left(\tau_{k, j}^{i+}\right)=\lambda_{S_{k}}\left(\tau_{k, j}^{i}\right) \\
& \lambda_{I_{k}^{1}}\left(\tau_{k, j}^{i+}\right)=\lambda_{I_{k}^{1}}\left(\tau_{k, j}^{i}\right) ; \\
& \lambda_{I_{k}^{2}}\left(\tau_{k, j}^{i+}\right)=\lambda_{I_{k}^{2}}\left(\tau_{k, j}^{i}\right) ; \\
& \lambda_{R_{k}}\left(\tau_{k, j}^{i+}\right)=\lambda_{R_{k}}\left(\tau_{k, j}^{i}\right) .
\end{aligned}
$$

Here is the conditions for $\Delta_{i}$ for each $I_{k}^{i}$ from the Theorem 1:

$$
\begin{aligned}
\Delta_{1}= & f_{k}^{1}\left(I_{k}^{1}\left(\tau_{k, j}^{1}\right)\right)-f_{k}^{1}\left(I_{k}^{1}\left(\tau_{k, j}^{1+}\right)\right)-g\left(R_{k}\left(\tau_{k, j}^{1}\right)\right)+g\left(R_{k}\left(\tau_{k, j}^{1+}\right)\right)+ \\
& c_{k, j}^{1}\left[\frac{d g\left(R_{k}\left(\tau_{k, j}^{1+}\right)\right)}{d R_{k}\left(\tau_{k, j}^{1+}\right)}+\frac{d f_{k}^{1}\left(I_{k}^{1}\left(\tau_{k, j}^{1+}\right)\right)}{d I_{k}^{1}\left(\tau_{k, j}^{1+}\right)}\right]+2 \sigma_{k}^{1} c_{k, j}^{1}\left(\lambda_{R_{k}}\left(\tau_{k, j}^{1+}\right)-\lambda_{I_{k}^{1}}\left(\tau_{k, j}^{1+}\right)\right)+ \\
& \delta_{1 k} S_{k}\left(\tau_{k, j}^{1}\right) c_{k, j}^{1}\left(\lambda_{S_{k}}\left(\tau_{k, j}^{1}\right)-\lambda_{I_{k}^{1}}\left(\tau_{k, j}^{1}\right)\right)\left[2 \Theta_{1}\left(\tau_{k, j}^{1+}\right)+\frac{\delta_{1 k} P(k)}{\langle k\rangle}\left(1+I_{k}^{1}\left(\tau_{k, j}^{1}\right)-c_{k, j}^{1}\right)\right] .
\end{aligned}
$$

Here is the conditions for $\Delta$ for each $\Delta$ for each $I_{k}^{2}$ from the Theorem 1 :

$$
\begin{aligned}
\Delta_{2}= & f_{k}^{2}\left(I_{k}^{2}\left(\tau_{k, j}^{2}\right)\right)-f_{k}^{2}\left(I_{k}^{2}\left(\tau_{k, j}^{2+}\right)\right)-g\left(R_{k}\left(\tau_{k, j}^{2}\right)\right)+g\left(R_{k}\left(\tau_{k, j}^{2+}\right)\right)+ \\
& c_{k, j}^{2}\left[\frac{d g\left(R_{k}\left(\tau_{k, j}^{2+}\right)\right)}{d R_{k}\left(\tau_{k, j}^{2+}\right)}+\frac{d f_{k}^{2}\left(I_{k}^{2}\left(\tau_{k, j}^{2+}\right)\right)}{d I_{k}^{2}\left(\tau_{k, j}^{2+}\right)}\right]+2 \sigma_{k}^{2} c_{k, j}^{2}\left(\lambda_{R_{k}}\left(\tau_{k, j}^{2+}\right)-\lambda_{I_{k}^{2}}\left(\tau_{k, j}^{2+}\right)\right)+ \\
& \delta_{2 k} S_{k}\left(\tau_{k, j}^{2}\right) c_{k, j}^{2}\left(\lambda_{S_{k}}\left(\tau_{k, j}^{2}\right)-\lambda_{I_{k}^{2}}\left(\tau_{k, j}^{2}\right)\right)\left[2 \Theta_{2}\left(\tau_{k, j}^{2+}\right)+\frac{\delta_{2 k} P(k)}{\langle k\rangle}\left(1+I_{k}^{2}\left(\tau_{k, j}^{2}\right)-c_{k, j}^{2}\right)\right] .
\end{aligned}
$$

According to Theorem 1 at time $\tau_{k, j}^{i} \in(0, T) \Delta_{i}$ should be equal to zero. Therefore, we deal with two different problems: firstly, if the intensity of impulses $c_{k, j}^{i}$ are fixed, then from (35) and (36), we can find the optimal time $\tau_{k, j}^{i *}$ of using impulses; secondly, if the sequence of time $\tau_{k, j}^{i}$ are fixed, then we obtain the optimal level of the intensity of impulses $c_{k, j}^{i *}, j=1, \ldots, q_{i}, i=1,2$.

\section{Numerical simulation of SIIR model with impulse control}

In the current section, we represent numerical experiments to depict theoretical results on the impulse controls structure. Here as in Experiment 1.4, we use the following set of the initial states and values of parameters of the system (22): initial system states and parameters are $S_{k}(0)=0.5, I_{k}^{1}(0)=0.3, I_{k}^{2}(0)=0.2$ and $R_{k}(0)=0$, spreading rates are $\delta_{1 k}=0.075 k, \delta_{2 k}=0.1 k$, self-recovery rates are $\sigma_{k}^{1}=0.0005 k$ and $\sigma_{k}^{1}=0.0003 k$. We set costs functions for infectious subgroups as $f_{k}^{i}\left(I_{k}^{i}(t)\right)=A_{k}^{i} I_{k}^{i}(t)$ with coefficients $A_{k}^{1}=2 k, A_{k}^{2}=3 k$ and treatment costs functions as $h_{k}^{i}\left(\nu_{k, j}^{i}\left(\tau_{k, j}^{i+}\right)=B_{k}^{i} c_{k, j}^{i} I_{k}^{i}\left(\tau_{k, j}^{i+}\right)\right.$, where coefficients are equal to $B_{k}^{1}=3 k$, $B_{k}^{2}=4 k, c_{k, j}^{1}=0.1, c_{k, j}^{2}=0.08$ for $i=1,2$, utility function is $g\left(R_{k}(t)\right)=0.1 R_{k}(t)$.

Experiment 3.1. We present the initial example of the behavior of the system and aggregated system costs in special case, when an average number of links between $i$-th node and its neighbours is $k=4$. Figs. 16 represent the propagation of two types of virus (left) and corresponding total system costs (right).

Aggregated system costs in this experiment are equal to $J=37.65$ (controlled case). By applying the control impulses at discrete time moments we received that a number of impulses are equal to $p_{1}(4)=37$ and $p_{2}(4)=49$. Comparing the aggregated system costs in impulse and continuous cases, we receive that value of functional $J$ are less in impulse formulation of the optimal control problem. 

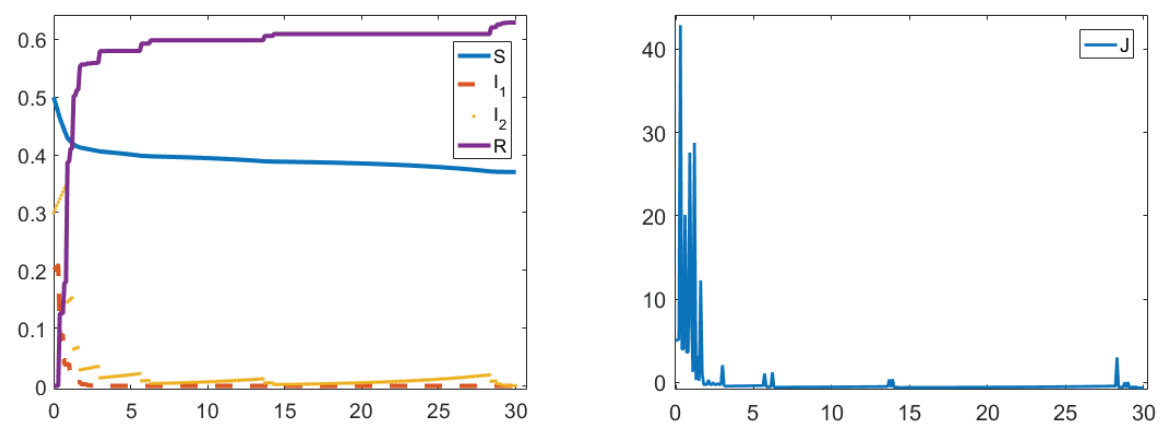

Fig. 16. Experiment 3.1. Left: Evolution of the system. Number of links: $k=4$, spreading rates: $\delta_{1 k}=0.075 k$ and $\delta_{2 k}=0.1 k$. Right: Aggregated system costs are equal to $J=37.65$.

Experiment 3.2. In this experiment we use the same parameters for initial data, but in contrast to Experiment 3.1 an average number of neighbours is equal to $k=7$.

We obtain that the aggregated costs are $J=73.93$ (controlled case), and an amount of impulses are equal to $p_{1}(7)=29$ and $p_{2}(7)=44$. We may notice that increasing the number of neighbour links increases the costs of the system. Since there are less nodes with connectivity $k=7$ which is more than average connectivity $\langle k\rangle=4$, we need less impulse treatment to vaccinate the network, thereby if we apply control to more connected nodes we reduce the costs of treatment.
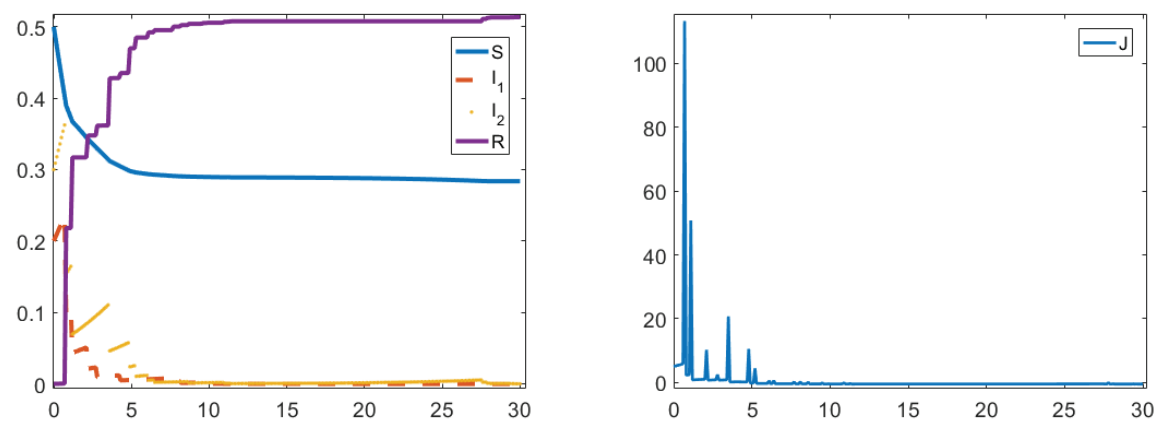

Fig. 17. Experiment 3.2. Left: Evolution of the system. Number of links: $k=7$, spreading rates: $\delta_{1 k}=0.075 k$ and $\delta_{2 k}=0.1 k$. Right: Aggregated system costs are equal to $J=73.93$.

Experiment 3.3. By using the same initial set of data we variate the spreading rate for virus and consider $\delta_{1 k}=0.1 k$ and $\delta_{2 k}=0.2 k$. Here we receive that the aggregated costs are $J=122.27$ and a number of impulses are $p_{1}(4)=43$ and $p_{2}(4)=55$, then increasing the spreading rates are leading to increasing aggregated costs and number of impulses which are needed to heal the network. 

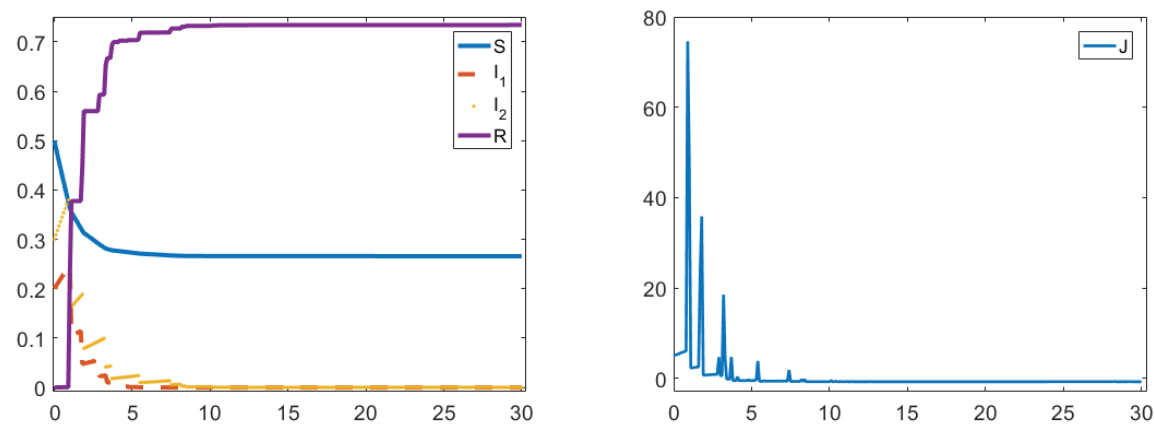

Fig. 18. Experiment 3.3. Left: Evolution of the system. Number of links: $k=4$, spreading rates: $\delta_{1 k}=0.1 k$ and $\delta_{2 k}=0.2 k$. Right: Aggregated system costs are equal to $J=122.27$.

\section{Conclusion}

This survey considers the optimal control problem in three modifications of the epidemic model. Firstly, we represent the model of two simultaneously spreading viruses and formulate the continuous control problem, secondly, the network formulation extends the SIIR model. It has been investigated the continuous and impulse optimal control problems for epidemic models of two coexisting types of viruses for heterogeneous populations, where control efforts are exerted at discrete time points, modelling the periodical scheduling of patching processes. We have obtained the structure of the optimal impulse controller as well as the form of continuous controls for a special class of cost functions. Numerical examples have been used to corroborate the theoretical results. From a comparison of the aggregated system costs in all sections, we have received that functional $J$ is higher in a continuous case on a fully connected network of Section 4 . This fact can be explained by the impact of networks structure on the population. If each node is connected to the neighbor node, then the virus spreads much faster, which leads to an increase in the total costs. However, this modification of the SIIR model does not require large computational costs and can be used to evaluate real-life situations. Models with continuous and impulse control over complex networks (Sections 6. and 9.) give approximately identical aggregated costs. However, in the impulse control model, numerical simulations showed that at the beginning of the epidemic process, the healthcare system might be more occupied. It means that more impulses are necessary to protect the system. From the economic point of view, this implies that it needs to treat more people at the beginning of the epidemic and provoke a collapse of the system. While in the continuous case, the control strategies are applied more uniformly, and it permits avoiding the overload of the healthcare system.

\section{Appendix 1.}

Define the associated Hamiltonian $H$ as follows: 


$$
\begin{aligned}
H= & f_{1}\left(I_{k}^{1}(t)\right)+f_{2}\left(I_{k}^{2}(t)\right)-g\left(R_{k}(t)\right)+h_{1}\left(u_{1}(t)\right)+h_{2}\left(u_{2}(t)\right)+ \\
& \left(\lambda_{I_{k}^{1}}(t)-\lambda_{S_{k}}(t)\right) \delta_{1} S_{k}(t) I_{k}^{1}(t) \Theta_{1}+\left(\lambda_{I_{k}^{2}}(t)-\lambda_{S_{k}}(t)\right) \delta_{2} S_{k}(t) I_{k}^{2}(t) \Theta_{2}+ \\
& \left(\lambda_{R_{k}}(t)-\lambda_{I_{k}^{1}}(t)\right) \sigma_{1} I_{k}^{1}(t)+\left(\lambda_{R_{k}}(t)-\lambda_{I_{k}^{2}}(t)\right) \sigma_{2} I_{k}^{2}(t)- \\
& \left(\lambda_{I_{k}^{1}}(t)-\lambda_{R_{k}}(t)\right) I_{k}^{1}(t) u_{1}(t)-\left(\lambda_{I_{k}^{2}}(t)-\lambda_{R_{k}}(t)\right) I_{k}^{2}(t) u_{2}(t) .
\end{aligned}
$$

We construct the associated adjoint system as follows:

$\dot{\lambda}_{S_{k}}(t)=-\left(\lambda_{I_{k}^{1}}-\lambda_{S_{k}}\right) \delta_{1} I_{k}^{1} \Theta_{1}-\left(\lambda_{I_{k}^{2}}-\lambda_{S_{k}}\right) \delta_{2} I_{k}^{2} \Theta_{2} ;$

$\dot{\lambda}_{I_{k}^{1}}(t)=-f_{1}^{\prime}\left(I_{k}^{1}\right)+\left(\lambda_{S_{k}}-\lambda_{I_{k}^{1}}\right) \delta_{1} S_{k} \Theta_{1}-\left(\lambda_{R_{k}}-\lambda_{I_{k}^{1}}\right) \sigma_{1}+\left(\lambda_{I_{k}^{1}}-\lambda_{R_{k}}\right) u_{1}$;

$\dot{\lambda}_{I_{k}^{2}}(t)=-f_{2}^{\prime}\left(I_{k}^{2}\right)+\left(\lambda_{S_{k}}-\lambda_{I_{k}^{2}}\right) \delta_{2} S_{k} \Theta_{2}-\left(\lambda_{R_{k}}-\lambda_{I_{k}^{2}}\right) \sigma_{2}+\left(\lambda_{I_{k}^{2}}-\lambda_{R_{k}}\right) u_{2} ;$

$\dot{\lambda}_{R_{k}}(t)=g^{\prime}\left(R_{k}\right)$;

with the transversality conditions given by

$$
\lambda_{I_{k}^{1}}(T)=0, \lambda_{I_{k}^{2}}(T)=0, \lambda_{S_{k}}(T)=0, \lambda_{R_{k}}(T)=0 .
$$

Following the Pontryagin's maximum principle (Pontryagin, 1987), there exist continuous and piecewise continuously differentiable co-state functions $\lambda_{i}$ that at every point $t \in[0, T]$ where $u_{1}$ and $u_{2}$ is continuous, satisfying (38) and (39). In addition, we have

$$
\left(u_{1}, u_{2}\right) \in \arg \min _{\underline{u}_{1}, \underline{u}_{2} \in[0,1]} H\left(\bar{\lambda},\left(S_{k}, I_{k}^{1}, I_{k}^{2}, R_{k}\right),\left(\underline{u}_{1}, \underline{u}_{2}\right)\right) .
$$

Define switching functions $\varphi_{k}^{i}$ as follows:

$$
\varphi_{k}^{1}=\left(\lambda_{I_{k}^{1}}-\lambda_{R_{k}}\right) I_{k}^{1}, \quad \varphi_{k}^{2}=\left(\lambda_{I_{k}^{2}}-\lambda_{R_{k}}\right) I_{k}^{2} .
$$

Based on previous research, e.g., (Altman, 2010; Pontryagin, 1987; Gubar, 2013), in this section, we show that an optimal control $u(t)=\left(u_{1}(t), u_{2}(t)\right)$ has following structure:

Functions $h_{i}(\cdot)$ and the Hamiltonian are concave

Let $h_{i}(\cdot)$ be a concave functions $\left(h_{i}^{\prime \prime}(\cdot)<0\right)$, then according to $(37)$, the Hamiltonian is a concave function of $u_{i}, i=\overline{1,2}$. There are two different options for $u_{i} \in[0,1]$ that minimize the Hamiltonian, i.e., if at the time moment $t$

$$
h_{i}(0)-\varphi_{k}^{i}(t) \cdot 0<h_{i}(1)-\varphi_{k}^{i}(t) \cdot 1,
$$

or

$$
h_{i}(1)>\varphi_{k}^{i}(t),
$$

then optimal control is $u_{i}=0$; otherwise $u_{i}=1$.

For $i=\overline{1,2}$, the optimal control parameters $u_{i}(t)$ are defined as follows:

$$
u_{i}^{*}(t)= \begin{cases}0, & \text { for } \quad \varphi_{k}^{i}(t)<h_{i}(1), \\ 1, & \text { for } \quad \varphi_{k}^{i}(t) \geq h_{i}(1) .\end{cases}
$$

Functions $\varphi_{k}^{i}$ are non-increasing functions, then there can be at most one time moment $t \in[0, T]$ at which $\varphi_{k}^{i}(t)=h_{i}^{\prime}\left(u_{i}(t)\right)$. Moreover, if such moment exists, for example, $t_{1}$, then $\varphi_{k}^{i}(t)<h_{i}(1)$ on $0 \leq t<t_{1}$ and $\varphi_{k}^{i}(t) \geq h_{i}(1)$ on $t_{1} \leq t<T$. 


\section{Functions $h_{i}(\cdot)$ and the Hamiltonian are strictly convex}

Let $h_{i}(\cdot)$ be a strictly convex functions $\left(h_{i}^{\prime \prime}(\cdot)>0\right)$, then Hamiltonian is a convex function. Consider the following derivative:

$$
\left.\frac{\partial}{\partial x}\left(h_{i}(x)-\varphi_{k}^{i} x\right)\right|_{x=x_{i}}=0,
$$

where $x_{i} \in[0,1], u_{i}^{*}(t)=x_{i}$. There are three different types of points at which the Hamiltonian reaches its minimum. To find them, we need to consider the derivatives of the Hamiltonian at $u_{i}=0$ and $u_{i}=1$. If the derivatives (42) at $u_{i}=0$ are increasing $\left(h_{i}^{\prime}(0)-\varphi_{k}^{i} \geq 0\right)$, then the value of the control that minimizes the Hamiltonian is less than 0 , and according to our restrictions $\left(u_{i} \in[0,1]\right)$ optimal control will be equal to 0 . If the derivatives at $u_{i}=1$ are non-increasing $\left(h_{i}^{\prime}(1)-\varphi_{k}^{i}<0\right)$, it means that the value of the control that minimizes the Hamiltonian is greater than 1. Hence the optimal control will be 1 ; otherwise, we can find such value $u_{i}^{*} \in(0,1)$.

Functions $\varphi_{k}^{i}(t), h_{i}^{\prime}(t), u_{i}^{*}(t)$ are continuous at all $t \in[0, T]$. In this case $h_{i}$ is strictly convex and $h_{i}^{\prime}$ is strictly increasing functions, so $h_{i}^{\prime}(0)<h_{i}^{\prime}(1)$. Thus there exist points $t_{0}$ and $t_{1}\left(0<t_{0}<t_{1}<T\right)$ so that conditions (43) are satisfied, and according to $\varphi_{k}^{i}(t)$ are non-decreasing functions. That is:

$$
u_{i}^{*}(t)= \begin{cases}0, & \text { for } \varphi_{k}^{i}(t) \leq h_{i}^{\prime}(0) \\ h^{\prime-1}\left(\varphi_{k}^{i}\right), & \text { for } h_{i}^{\prime}(0)<\varphi_{k}^{i}(t) \leq h_{i}^{\prime}(1) \\ 1, & \text { for } h_{i}^{\prime}(1)<\varphi_{k}^{i}(t) .\end{cases}
$$

\section{Appendix 2.}

Define the associated Hamiltonian $H$ as follows:

$$
\begin{aligned}
H= & f_{1}\left(I_{k}^{1}(t)\right)+f_{2}\left(I_{k}^{2}(t)\right)+h_{1}\left(u_{1}(t)\right)+h_{2}\left(u_{2}(t)\right)-g\left(S_{k}(t)\right)+ \\
& \lambda_{S_{k}}(t)\left(-l_{1} S_{k}(t) \Theta_{1}(t)-l_{2} S_{k}(t) \Theta_{2}(t)+u_{1} I_{k}^{1}(t)+u_{2} I_{k}^{2}(t)+I_{k}^{1}(t)+I_{k}^{2}(t)\right)+ \\
& \lambda_{I_{k}^{1}}(t)\left(l_{1} S(t) \Theta_{1}(t)-I_{k}^{1}(t)-u_{1} I_{k}^{1}(t)\right)+\lambda_{I_{k}^{2}}\left(l_{2} S_{k}(t) \Theta_{2}(t)-I_{k}^{2}(t)-u_{2} I_{k}^{2}(t)\right) .
\end{aligned}
$$

The adjoint system is equal to

$$
\begin{aligned}
& \dot{\lambda}_{S_{k}}(t)=-g^{\prime}(S)-\lambda_{S_{k}}\left(-l_{1} \Theta_{1} I_{k}^{1}-l_{2} \Theta_{2} I_{k}^{2}\right)-\lambda_{I_{k}^{1}} l_{1} \Theta_{1} I_{k}^{1}-\lambda_{I_{k}^{2}} l_{2} \Theta_{2} I_{k}^{2} \\
& \dot{\lambda}_{I_{k}^{1}}(t)=f_{1}^{\prime}\left(I_{k}\right)-\lambda_{S_{k}}\left(-l_{1} S_{k} \Theta_{1}+u_{1}+1\right)-\lambda_{I_{k}^{1}}\left(l_{1} S_{k} \Theta_{1}-1-u_{1}\right) \\
& \dot{\lambda}_{I_{k}^{2}}(t)=f_{2}^{\prime}\left(I_{k}\right)-\lambda_{S_{k}}\left(l_{2} S_{k} \Theta_{2}+u_{2}+1\right)-\lambda_{I_{k}^{2}}\left(l_{2} S_{k} \Theta_{2}-1-u_{2}\right) .
\end{aligned}
$$

with the transversality condition:

$$
\lambda_{I_{k}^{1}}(T)=0, \lambda_{I_{k}^{2}}(T)=0, \lambda_{S_{k}}(T)=0 .
$$

According to Pontryagin's maximum principle, there exist continuous and piecewise continuously differentiable co-state functions $\lambda_{i}$ that at every point $t \in[0, T]$ where $u$ is continuous, satisfy (44) and (45).

$$
\left(u_{1}, u_{2}\right) \in \arg \min _{\overline{u_{1}}, \overline{u_{2}} \in[0,1]} H\left(\lambda,(S, I, R), \overline{u_{1}}, \overline{u_{2}}\right),
$$




\section{References}

Agur, Z. et al. (1993). Pulse mass measles vaccination across age cohorts. Proceedings of the National Academy of Sciences of the United States of America, 90, 11698-11702.

Altman, E. et al. (2010). Dispatch then stop: Optimal dissemination of security patches in mobile wireless networks. In Proceedings of the 48th IEEE Conference on Decisions and Control (CDC). pp. 2354-2359.

Balter, M. (1998). New HIV strain could pose health threat. Science, 281(5382), 1425-1426.

Barabási, A. L. and R. Albert (1999). Emergence of scaling in random networks. Science, 286(5439), 509-512.

Barabási, A.-L. and R. Albert (2002). Statistical mechanics of complex networks. Rev. Mod. Phys., 74, 47.

Behncke, H. (2000). Optimal control of deterministic epidemics. Optimimal Control Appl. Methods, 21, 269-285.

Blaquière, A. (1985). Impulsive Optimal Control with Finite or Infinite Time Horizon. Journal Of Optimization Theory And Applications. Vol. 46.

Bomze, I. and S. Rota Bulò (2011). Infection and immunization: A new class of evolutionary game dynamics. Games Econ. Behav, 71, 193-211.

Bonhoeffer, S. et al. (1997). Virus dynamics and drug therapy. Proc. Natl. Acad. Sci. USA, 94, 6971-6976.

Butler, D. (2012). Flu surveillance lacking. Nature, 483, 520-522.

Capasso, V. (1993). Mathematical Structures of Epidemic Systems; Lecture Notes in Biomathematics; Springer: Berlin/Heidelberg, Germany. Vol. 97.

Castillo-Chavez, C. et al. (1996). Competitive exclusion in gonorrhea models and other sexually transmitted diseases. SIAM J. on App. Math., 56, 2, 494-508.

Chahim, M. et al. (2012). A tutorial on the deterministic Impulse Control Maximum Principle: Necessary and sufficient optimality conditions. European Journal of Operational Research, 219, 18-26.

Chowell, G. et al. (2017). Perspectives on model forecasts of the 2014-2015 Ebola epidemic in West Africa: Lessons and the way forward. BMC Med. Vol. 15, 42. doi:10.1186/s12916-017-0811-y.

Conn, M. (2006). Handbook of Models for Human Aging; Academic Press: London, UK.

Dykhta, V. A. and O. N. Samsonyuk (2009). A maximum principle for smooth optimal impulsive control problems with multipoint state constraints. Computational Mathematics and Mathematical Physics, 49, 942-957.

Francis, P. J. (2004). Optimal tax/subsidy combinations for the flu season. J. Econ. Dyn. Control, 28, 2037-2054.

Fu X. et al. (2008). Epidemic dynamics on scale-free networks with piecewise linear infectivity and immunization. Phys. Rev. E., 77, 3, 036113.

Fu, F. et al. (2011). Imitation dynamics of vaccination behaviour on social networks. Proc. R. Soc. Lond. Ser. B., 278, 42-49.

Gjorgjieva, J. et al. (2005). The role of vaccination in the control of SARS. Math. Biosci. Eng., 2, 753-769.

Gubar, E. and Q. Zhu (2013). Optimal Control of Influenza Epidemic Model with Virus Mutations. Proceedings of the 12th Biannual European Control Conference, Zurich, Switzerland. IEEE Control Systems Society: New York, NY, USA, 2013; pp. 31253130 .

Gubar E. et al. (2015). Impact of Propagation Information in the Model of Tax Audit. Recent Advances in Game Theory and Applications. "Static and Dynamic Game Theory: Foundations and Applications". Switzerland. pp. 91-110.

Kermack, W. O. and A. G. McKendrick (1927). A contribution to the mathematical theory of epidemics. Proceedings of the Royal Society of London. Series A. Vol. 115, 772, pp. 700-721. The Royal Society, New York. 
Kharraz, A. et al. (2015). Cutting the gordian knot: A look under the hood of ransomware attacks. International Conference on Detection of Intrusions and Malware, and Vulnerability Assessment. pp. 3-24. Springer.

Khatri, S. et al. (2003). The Role of Network Topology on the Initial Growth Rate of Influenza Epidemic. Technical Report BU-1643-M; published by KeAi, China.

Khouzani, M.H.R. et al. (2010). Maximum damage malware attack mobile wireless networks. Proceedings of the 29th International Conference on Computer Communications (INFOCOM), San Diego, CA, USA. pp. 749-757.

Khouzani, M. H. R. et al. (2011). Optimal control of epidemic evolution. Proceedings of the 30th International Conference on Computer Communications (INFOCOM), Shanghai, China. pp. 1683-1691.

Leander, R. et al. (2015). Optimal control of continuous systems with impulse controls. Optimal Control Applications and Methods Optim. Control Appl. Meth., 36:-549.

Luo, X. and Q. Liao (2009). Ransomware: A new cyber hijacking threat to enterprises. Handbook of research on information security and assurance. 2009:1-6.

Masuda, N. and N. Konno (2006). Multi-state epidemic processes on complex networks. J. Theor. Biol., 243, 1, 64-75.

Newman, M. E. J. (2005). Threshold effects for two pathogens spreading on a network. Phys. Rev. Lett., 95, p. 108701.

Newman, L. H. (2016). What we know about Friday's massive east coast Internet outage. Wired Magazine.

Nuno, M. et al. (2005). Dynamics of two-strain influenza with isolation and partial crossimmunity. SIAM J. on App. Math., 65, 3, 964-982.

Omic, J. et al. (2009). Protecting against network infections: A game theoretic perspective. Proceedings of the 28th IEEE Conference on Computer Communications (INFOCOM), Rio de Janeiro, Brazil.

Pappas, G. J. et al. (2016). Analysis and Control of Epidemics: A Survey of Spreading Processes on Complex Networks, in IEEE Control Systems Magazine, 36(1), 26-46. doi: 10.1109/MCS.2015.2495000.

Pastor-Satorras, R. and A. Vespignani (2001). Epidemic spreading in scale-free networks. Phys. Rev. Lett., 86, 14, 3200.

Perkins, T.A. and G. Espana (2020). Optimal Control of the COVID-19 Pandemic with Non-pharmaceutical Interventions. Bulletin of mathematical biology 82(9), 118. https://doi.org/10.1007/s11538-020-00795-y.

Pontryagin, L. S. (1987). Mathematical theory of optimal processes. CRC Press.

Pragyan, D. et al. (2020). The Economic Effects of Covid-19 Containment Measures. IMF Working Paper WP/20/158.

Rohloff, K. R. and T. Başar (2008). Deterministic and stochastic models for the detection of random constant scanning worms. ACM Trans. Model. Comput. Simul. (ACM TOMACS), 18, 1-24.

Rowson, T. et al. (2020). How and When to End the COVID-19 Lockdown: an Optimization approach. Frontiers of Public Health, 10. https://doi.org/10.3389/fpubh.2020.00262.

Rowthorn, R. and F. Toxvaerd (2020). The Optimal Control of Infectious Diseases via Prevention and Treatment. Technical Report 2013, Cambridge-INET Working Paper.

Sethi, S. P. and G. L. Thompson (2006). Optimal Control Theory: Applications to Management Science and Economics. Springer, Berlin.

Smith, G. et al. (2009). Origins and evolutionary genomics of the 2009 swine-origin H1N1 influenza A epidemic. Nature, 459, 7250, 1122-1125.

Strogatz, S. H. (2001). Exploring complex networks. Nature, 410, 6825, 268-276.

Taynitskiy, V., Gubar, E. and E. Zhitkova (2015). Structure of optimal control in the model of propagation of two malicious softwares. Proceedings of the International Conference "Stability and Control ProcessesвЂ⿳́ in Memory of V.I. Zubov (SCP), SaintPetersburg, Russia. pp. 261-264. 
Taynitskiy, V., Gubar, E. and Q. Zhu (2016). Optimal Security Policy for Protection Against Heterogeneous Malware. Proceedings of the International Conference on вЂњNetwork Games, Control and OptimizationвЂќ (NETGCOOP 2016), Avignon, France. pp. 199-210.

Taynitskiy, V., Gubar, E. and E. Zhitkova (2016). Optimization of protection of computer networks against malicious software. Proc. of International Conference "Stability and Oscillations of Nonlinear Control Systems" (Pyatnitskiy's Conference).

Taynitskiy, V., Gubar, E. and Q. Zhu (2017). Optimal Impulse Control of Bi-Virus SIR Epidemics with Application to Heterogeneous Internet of Things. Constructive Nonsmooth Analysis and Related Topics. Abstracts of the International Conference. Dedicated to the Memory of Professor V.F. Demyanov. pp. 113-116.

Taynitskiy, V., Gubar, E. and Q. Zhu (2017). Optimal Control of Multi-strain Epidemic Processes in Complex Networks. Game Theory for Networks. GameNets 2017. Lecture Notes of the Institute for Computer Sciences, Social Informatics and Telecommunications Engineering. Vol 212. Springer, Cham. pp. 108-117.

Thomasey, D. H. and M. Martcheva (2008). Serotype replacement of vertically transmitted diseases through perfect vaccination. J. Biol. Sys., 16, 02, 255-277.

Vespignani, A. et al. (2015). Epidemic processes in complex networks. Rev. Mod. Phys., 87, 925-979.

Wickwire, K. H. (1975). A note on the optimal control of carrier-borne epidemics. J. Appl. Probab., 12, 565-346.

Zaccour, G., Reddy, P. and S. Wrzaczek (2016). Quality effects in different advertising models - An impulse control approach. European Journal of Operational Research, 255, 984-995.

Zhu, Q., Bushnell, L. and T. Başar (2012). Game-theoretic analysis of node capture and cloning attack with multiple attackers in wireless sensor networks. In Proceedings of the 51st IEEE Conference on Decision and Control (CDC'12), Maui, HI, USA. 